\title{
Moduli spaces of algebras over nonsymmetric operads
}

\author{
FERNANDO MURO
}

In this paper we study spaces of algebras over an operad (nonsymmetric) in symmetric monoidal model categories. We first compute the homotopy fiber of the forgetful functor sending an algebra to its underlying object, extending a result of Rezk. We then apply this computation to the construction of geometric moduli stacks of algebras over an operad in a homotopical algebraic geometry context in the sense of Toën and Vezzosi. We show under mild hypotheses that the moduli stack of unital associative algebras is a Zariski open substack of the moduli stack of nonnecessarily unital associative algebras. The classical analogue for finite-dimensional vector spaces was noticed by Gabriel.

18D50, 14K10; 55U35

\section{Introduction}

Let $\mathbb{k}$ be a commutative ring. An associative algebra structure on a free $\mathbb{k}$-module $F$ of rank $n$ with basis $\left\{e_{1}, \ldots, e_{n}\right\} \subset F \cong \mathbb{k}^{n}$ is determined by structure constants $c_{i j}^{k}$, $1 \leq i, j, k \leq n$, such that

$$
e_{i} \cdot e_{j}=\sum_{k=1}^{n} c_{i j}^{k} e_{k}
$$

The associativity condition $\left(e_{i} \cdot e_{j}\right) \cdot e_{k}=e_{i} \cdot\left(e_{j} \cdot e_{k}\right)$ is equivalent to the identities of structure constants

$$
\sum_{m=1}^{n} c_{i j}^{m} c_{m k}^{l}=\sum_{m=1}^{n} c_{i m}^{l} c_{j k}^{m}, \quad 1 \leq l \leq n .
$$

Therefore, the moduli space of associative algebra structures on a free module of rank $n$ is the finitely presented affine subspace $\operatorname{Spec} R \subset \mathbb{A}^{n^{3}}$,

$$
R=\mathbb{k}\left[c_{i j}^{k} ; 1 \leq i, j, k \leq n\right] /\left(\sum_{m=1}^{n}\left(c_{i j}^{m} c_{m k}^{l}-c_{i m}^{l} c_{j k}^{m}\right) ; 1 \leq i, j, k, l \leq n\right) .
$$


A unital associative algebra structure on $F$ is given by an associative algebra structure together with a unit element

$$
\mathbf{1}=\sum_{i=1}^{n} a_{i} e_{i} \in F
$$

satisfying the following equations for $1 \leq k \leq n$, which are equivalent to $\mathbf{1} \cdot e_{i}=e_{i}=e_{i} \cdot \mathbf{1}$, $1 \leq i \leq n$ :

$$
\begin{aligned}
&\left(a_{1}, \ldots, a_{n}\right)\left(\begin{array}{ccc}
c_{11}^{k} & \cdots & c_{1 n}^{k} \\
\vdots & \ddots & \vdots \\
c_{n 1}^{k} & \cdots & c_{n n}^{k}
\end{array}\right)=(0, \ldots, 1, \ldots, 0) \\
&\left(\begin{array}{ccc}
c_{11}^{k} & \cdots & c_{1 n}^{k} \\
\vdots & \ddots & \vdots \\
c_{n 1}^{k} & \cdots & c_{n n}^{k}
\end{array}\right)\left(\begin{array}{c}
a_{1} \\
\vdots \\
a_{n}
\end{array}\right)=\left(\begin{array}{c}
0 \\
\vdots \\
1 \\
\vdots \\
0
\end{array}\right) \leftarrow k^{\text {th } \text { place }}
\end{aligned}
$$

Hence, the moduli space of unital associative algebra structures on a free module of rank $n$ is the affine subspace $\operatorname{Spec} S \subset \mathbb{A}^{n^{3}+n}$,

$$
S=R\left[a_{1}, \ldots, a_{n}\right] /\left(\delta_{j k}-\sum_{i=1}^{n} a_{i} c_{i j}^{k}, \delta_{j k}-\sum_{i=1}^{n} c_{j i}^{k} a_{i} ; 1 \leq j, k \leq n\right),
$$

where $\delta_{k k}=1$ and $\delta_{j k}=0$ if $j \neq k$.

The morphism $f: \operatorname{Spec} S \rightarrow \operatorname{Spec} R$ consisting of forgetting the unit is induced by the inclusion $R \subset S$. This morphism is a categorical monomorphism since an associative algebra may have at most one unit. Moreover, $f$ is a Zariski open immersion, ie it is also flat and of finite presentation; see Gabriel [12, 2.1 Lemma] and CrawleyBoevey [8, page 4].

Associative algebra structures are somewhat rigid. We are rather interested in them up to isomorphism. The algebraic group $\operatorname{GL}_{n} \cong \operatorname{Aut}_{\mathbb{k}}(F)$ acts on $\operatorname{Spec} R$. The orbits are the isomorphism classes of associative algebra structures. The isotropy group at a given point is the automorphism group of the corresponding associative algebra structure.

In order to obtain a meaningful quotient which remembers all this, we must move to the category of algebraic stacks. The quotient stack

$$
\underline{\operatorname{Ass}}_{n}=\operatorname{Spec} R / \mathrm{GL}_{n}
$$


is the moduli stack of associative algebras on rank $n$ vector bundles. The same applies to $\operatorname{Spec} S$, and the quotient

$$
\underline{\mathrm{uAss}}_{n}=\operatorname{Spec} S / \mathrm{GL}_{n}
$$

is the moduli stack of unital associative algebras on rank $n$ vector bundles. The morphism $f:$ Spec $S \rightarrow \operatorname{Spec} R$ induces a morphism between these algebraic stacks,

$$
\underline{f:} \underline{\mathrm{uAss}}_{n} \longrightarrow \underline{\mathrm{Ass}}_{n},
$$

which inherits most properties from $f$, eg $f$ is a monomorphism, affine, locally of finite presentation and flat; see Dwyer and Kan [15].

These stacks can be described as follows. The category Aff $\mathbb{\mathbb { k }}_{\mathbb{k}}$ of affine schemes over $\mathbb{k}$ is opposite to the category of commutative (associative and unital) $\mathbb{k}$-algebras. We assume this category is endowed with the étale topology. For any commutative $\mathbb{k}$-algebra $A$, let $\operatorname{Ass}_{n}(A)$ be the category of associative $A$-algebras whose underlying $A$-module is locally free of rank $n$. Denote by $i \operatorname{Ass}_{n}(A)$ the subcategory of isomorphisms. A change of coefficient functors gives rise to a pseudofunctor

$$
\begin{gathered}
\operatorname{Aff}_{\mathbb{k}}^{\text {op }} \longrightarrow \text { Groupoids, } \\
A \mapsto i \operatorname{Ass}_{n}(A),
\end{gathered}
$$

which is the 'functor of points' of the stack $\underline{\operatorname{Ass}}_{n}$. Similarly, if $\mathrm{uAss}_{n}(A)$ is the category of unital associative $A$-algebras whose underlying $A$-module is locally free of rank $n$, the 'functor of points' of $\underline{\mathrm{uAss}} n$ is

$$
\begin{gathered}
\operatorname{Aff}_{\mathbb{k}}^{\text {op }} \longrightarrow \text { Groupoids, } \\
A \mapsto i \operatorname{uAss}_{n}(A),
\end{gathered}
$$

and the morphism $f: \underline{\mathrm{uAss}}_{n} \rightarrow \underline{\mathrm{Ass}}_{n}$ is induced by the functors forgetting the unit $\mathrm{uAss}_{n}(A) \rightarrow \operatorname{Ass}_{n}(\bar{A})$.

In this paper we consider the same situation in a homotopical algebraic geometry (HAG) context in the sense of Toën and Vezzosi [26]. In such a context, the category of $\mathbb{k}$-modules is replaced with a symmetric monoidal model category $\mathscr{V}$ and the category of affine schemes Aff $\mathscr{V}$ is the opposite of the category of commutative algebras (ie monoids) in $\mathscr{V}$. There is also a fixed model pretopology $\tau$ and a class $\boldsymbol{P}$ of morphisms which plays the role of smooth morphisms. A stack is a contravariant functor from affine schemes to simplicial sets, $\mathrm{Aff}_{\mathscr{V}}^{\mathrm{op}} \rightarrow \mathrm{Set}^{\Delta^{\mathrm{op}}}$, satisfying certain homotopy invariance and descent properties. 
Given an operad $\mathcal{O}$ in $\mathscr{V}$ (nonsymmetric), admissible in the sense of Definition 4.1, we define a stack $\operatorname{Alg}_{\mathcal{V}}(\mathcal{O})$ such that, for any commutative algebra $A$,

$$
\underline{\operatorname{Alg}}_{V}(\mathcal{O})(A) \simeq\left|w \operatorname{Alg}_{\operatorname{Mod}(A)}(\mathcal{O})\right|
$$

is the classifying space of the category of weak equivalences between $\mathcal{O}$-algebras in the category $\operatorname{Mod}(A)$ of $A$-modules. We call $\operatorname{Alg}_{V}(\mathcal{O})$ the moduli stack of $\mathcal{O}$-algebras.

Notice that we proceed in a way inverse to the classical situation illustrated above. We do not present $\operatorname{Alg}_{\mathscr{V}}(\mathcal{O})$ as a quotient of an affine stack, but in terms of its 'functor of points'. The connection to affine stacks is described below.

Admissibility is not a very strong condition since all cofibrant operads are admissible. Moreover, the associative and unital associative operads, Ass and uAss, are always admissible.

The stack of quasicoherent modules is a stack QCoh such that, for any commutative algebra $A$,

$$
\underline{\mathrm{QCoh}}(A) \simeq|w \operatorname{Mod}(A)|
$$

is the classifying space of the category of weak equivalences between $A$-modules. We show that the forgetful functors from $\mathcal{O}$-algebras to modules gives rise to a morphism of stacks

$$
\xi^{\mathcal{O}}: \underline{\operatorname{Alg}_{V}}(\mathcal{O}) \longrightarrow \underline{\text { QCoh}} .
$$

The stack QCoh is too big and one is often interested in smaller substacks satisfying nice geometric properties, such as the stack Vect $_{n}$ of rank $n$ vector bundles, or the stack Perf of perfect modules; see [26, Section 1.3.7]. We will consider a generic substack $F \subset \underline{Q}$ Coh such that the connected components of $F(A)$ are represented by perfect $A$-modules, and often restrict to the substack $\operatorname{Alg}_{F}(\mathcal{O}) \subset \operatorname{Alg}_{\mathscr{V}}(\mathcal{O})$ obtained as the homotopy pullback of $\xi^{\mathcal{O}}$ along the inclusion $\overline{F \subset \underline{Q C o h}}$.

We prove that the restriction

$$
\xi_{F}^{\mathcal{O}}: \underline{\operatorname{Alg}_{F}}(\mathcal{O}) \longrightarrow F
$$

is an affine morphism (Theorem 5.17). The homotopy fiber of $\xi^{\mathcal{O}}$ at an $A$-point $\mathbb{R S p e c}(A) \rightarrow F$ represented by a perfect $A$-module $M$ is an affine stack over $\mathbb{R} \overline{\operatorname{Spec}}(A)$

$$
\underline{\operatorname{Map}}_{\mathrm{Op}(\mathscr{V})}\left(\mathcal{O}, \operatorname{End}_{\operatorname{Mod}(A)}(M)\right)
$$

called moduli stack of $\mathcal{O}$-algebra structures on $M$, since, for any $A$-algebra $B$,

$$
\underline{\operatorname{Map}}_{\mathrm{Op}(\mathscr{V})}\left(\mathcal{O}, \operatorname{End}_{\operatorname{Mod}(A)}(M)\right)(B) \simeq \operatorname{Map}_{\mathrm{Op}(\mathscr{V})}\left(\mathcal{O}, \operatorname{End}_{\operatorname{Mod}(B)}\left(\widetilde{M \otimes_{A}^{\mathbb{L} B}}\right)\right)
$$


is the mapping space in the model category $\operatorname{Op}(\mathscr{V})$ of operads in $\mathscr{V}$ from $\mathcal{O}$ to the endomorphism operad of a fibrant-cofibrant replacement of the $B$-module $M \otimes_{A}^{\mathbb{L}} B$, so it does classify derived $\mathcal{O}$-algebra structures on derived extensions of scalars of $M$.

A consequence of this fact is that $\operatorname{Alg}_{F}(\mathcal{O})$ is geometric provided $F$ is, eg $F=\underline{\text { Vect }}_{n}$ in many HAG contexts, and $F=\overline{\text { Perf }}$ in the weak complicial and brave new algebraic geometry contexts. Notice that

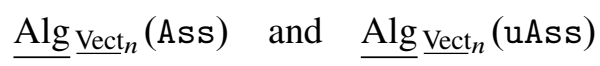

are the immediate generalizations of $\underline{\mathrm{Ass}_{n}}$ and $\underline{\mathrm{uAss}} n$ above.

We finally tackle our goal. The stack $\operatorname{Alg}_{V}(\mathcal{O})$ is a contravariant functor in $\mathcal{O}$. The operad morphism $\phi$ : Ass $\rightarrow$ uAss which models the forgetful functor from unital associative algebras to associative algebras induces a morphism of stacks

$$
\underline{\operatorname{Alg}_{V}}(\phi): \underline{\operatorname{Alg}} \mathscr{V}(\mathrm{uAss}) \longrightarrow \underline{\operatorname{Alg}_{V}}(\text { Ass }),
$$

which generalizes $\underline{f}$ above.

Similarly, $\phi$ induces a morphism of affine stacks

$$
\underline{\operatorname{Map}}_{\mathrm{Op}(V)}\left(\mathrm{uAss}, \operatorname{End}_{\operatorname{Mod}(A)}(M)\right) \longrightarrow \underline{\operatorname{Map}} \underline{\operatorname{Mp}(\mathscr{V})}\left(\operatorname{Ass}, \operatorname{End}_{\operatorname{Mod}(A)}(M)\right),
$$

which generalizes $f$.

Our main results on the properties of these morphisms are summarized in the following theorem. We refer the reader to [26] for the definition of the geometric terms in the statement.

Theorem 1.1 Consider the morphism $\operatorname{Alg}_{F}(\phi): \underline{\operatorname{Alg}}_{F}$ (uAss) $\rightarrow \operatorname{Alg}_{F}$ (Ass) obtained by restricting (1-1). Let $M$ be a perfect $\bar{A}$-module.

(1) The morphism $\operatorname{Alg}_{F}(\phi)$ is affine, ie (-1)-representable. The morphism (1-2) is obviously affine since it has affine source and target.

(2) If $\mathscr{V}$ is simplicial or complicial, then (1-1) and (1-2) are monomorphisms of

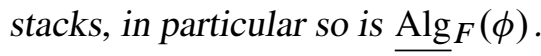

(3) If in addition $\mathscr{V}$ is locally finitely presentable as a category, finitely generated as a model category, and the tensor unit is finitely presented, then (1-2) is a finitely presented morphism of affine stacks and $\operatorname{Alg}_{F}(\phi)$ is categorically locally finitely presented. 
The crucial tool in the proof of (1) is a nonsymmetric generalization of one of the main theorems in Rezk's thesis [22, Theorem 1.1.5], which computes the homotopy fibers of the forgetful functor sending an $\mathcal{O}$-algebra to the underlying object; see Theorem 4.6 below. The proof of (2) is based in the main theorem by the author in [19], which proves that $\phi$ is a homotopy epimorphism in $\operatorname{Op}(\mathscr{V})$. For the proof of (3) we use Lurie's [16, Theorem 5.2.3.5].

The only property of affine Zariski open immersions we do not address in Theorem 1.1 is flatness. Flatness is defined in arbitrary HAG contexts for morphisms between affine stacks, but otherwise it is only defined for morphisms between geometric stacks whenever flatness is a local property for $\tau$, so it may well happen that it does not make sense to speak about the flatness of $\operatorname{Alg}_{F}(\phi)$ in a given HAG context. This contrasts with the properties considered in Theorem 1.1, which are defined in all HAG contexts. Nevertheless, in most HAG contexts of interest $\mathscr{V}$ is stable, with the remarkable exception of the derived algebraic geometry context. In stable contexts, all morphisms of affine stacks are flat, hence flatness is a local property and all morphisms between geometric stacks are flat, so (1-2) and $\operatorname{Alg}_{F}(\phi)$ would be flat in this situation if $F$ were geometric.

The morphisms (1-2) and $\operatorname{Alg}_{F}(\phi)$ are also flat in derived algebraic geometry. Indeed, since (1-2) is a finitely presented monomorphism, it is étale by [26, Proposition 1.2.6.5], and therefore flat by [26, Corollary 2.2.2.11]. Flatness is a local property in this context by [26, Lemma 2.2.3.4], hence it easily follows that $\operatorname{Alg}_{F}(\phi)$ is flat in the sense of [26, Definition 1.3.6.2]; compare the proof of Proposition 6.15.

To finish, in contrast with the classical situation, we show with an example that the stack $\operatorname{Map}_{\mathrm{Op}(\mathscr{V})}\left(\operatorname{Ass}, \operatorname{End}_{\operatorname{Mod}(A)}(M)\right)$ need not be finitely presented. Our example is in complicial algebraic geometry, in the two HAG contexts considered in [26, Section 2.3]. It is simply given by $A=\mathbb{k}=\mathbb{Q}$ and $M=\Sigma^{n} \mathbb{Q}$ for $n \leq-2$.

Moduli stacks of algebras over operads have already been considered by Toën and Vezzosi in the following specific situations [25; 26].

In the derived algebraic geometry context, where $\mathscr{V}=\operatorname{Mod}(\mathbb{k})^{\Delta^{\text {op }}}$ is the category of simplicial modules over a commutative ring $\mathbb{k}$, the stack $\operatorname{Alg}_{\text {Vect }_{n}}(\mathcal{O})$ and the map $\xi_{\text {Vect }_{n}}^{\mathcal{O}}$ were considered in [26, Section 2.2.6.2] for $\mathcal{O}$ an operad of projective $\mathbb{k}$-modules regarded as a constant simplicial operad. They prove that $\xi_{\underline{\text { Vect }_{n}}}^{\mathcal{O}}$ is affine and hence $\operatorname{Alg}_{\mathrm{Vect}_{n}}(\mathcal{O})$ is geometric. In the proof, they use Rezk's aforementioned theorem in a very clever way.

The stack $\operatorname{Alg}{ }_{\text {Perf }}($ Ass $)$ and the map $\xi_{\text {Perf }}^{\text {Ass }}$ were also considered in the complicial algebraic geometry context [26, Sections 2.3.3.2 and 2.3.5.3] and in the brave new 
algebraic geometry context $[25$, Section 4.2$]$. In these contexts, $\mathscr{V}=\mathrm{Ch}(\mathbb{k})$ is the category of chain complexes over a commutative $\mathbb{Q}$-algebra $\mathbb{k}$ and the category of symmetric spectra, respectively. They invoke Rezk's Theorem to deduce that $\xi_{\text {Perf }}^{\text {Ass }}$ is affine and that $\operatorname{Alg}_{\text {Perf }}$ (Ass) is geometric.

Toën and Vezzosi, however, did not provide full proofs of these claims in [25; 26]. On the one hand, they leave the reader to check that these moduli stacks of algebras over operads satisfy the required homotopy invariance and descent properties. On the other hand, Rezk did not prove his theorem for $\mathrm{Ch}(\mathbb{k})$ or for the category of symmetric spectra, and it is not completely obvious how to do so. Certainly, Rezk's proof does not extend to $\mathrm{Ch}(\mathbb{k})$. For instance, he uses in a crucial way the simplicial structure of $\operatorname{Set}^{\Delta^{\mathrm{op}}}$ and $\operatorname{Mod}(\mathbb{k})^{\Delta^{\mathrm{op}}}$, but $\mathrm{Ch}(\mathbb{k})$ is the paradigm of model category which is not simplicial. However, the results and proofs in this paper subsume such gaps in $[25 ; 26]$.

When proving homotopy invariance and descent for $\operatorname{Alg}_{\mathcal{V}}(\mathcal{O})$ and that $\xi_{F}^{\mathcal{O}}$ is affine, and hence $\operatorname{Alg}_{F}(\mathcal{O})$ is geometric, we closely follow the ideas in [26]. Moreover, our proof of the generalization of Rezk's Theorem also follows [22] in different ways, in particular we use an extension of Quillen's Theorem B and some computations of classification complexes. Therefore, this paper is very much indebted to Rezk, Toën and Vezzosi.

The paper is structured as follows. In Section 2 we fix terminology concerning model categories and monoidal structures, and recall some notions about operads and their algebras. Section 3 considers homotopy invariance properties of endomorphism operads, which play a role in the proof of the generalization of Rezk's Theorem in Section 4.

The moduli stacks of algebras and algebra structures over an admissible operad are constructed in Section 5. The existence of $\operatorname{Alg}_{V}(\mathcal{O})$ is far from obvious. The proofs of invariance and descent properties depend heavily on the homotopy theory of nonsymmetric operads developed by the author in $[20 ; 21]$. Neither the existence of $\xi^{\mathcal{O}}$ nor the contravariant functoriality of $\operatorname{Alg}_{\mathcal{V}}(\mathcal{O})$ is obvious. We also prove in this section those properties of $\xi^{\mathcal{O}}$ and the moduli stacks of algebras and algebra structures which hold for any admissible operad $\mathcal{O}$.

Finally, in Section 6 we concentrate in $\mathcal{O}=$ Ass, uAss and in the maps (1-1) and (1-2). Theorem 1.1 follows directly from the results in that section.

At the end of the paper, there is a short appendix on a technical property of the tensor unit of a monoidal model category, which is always satisfied by the underlying symmetric monoidal model category $\mathscr{V}$ of a HAG context. It is also satisfied when the tensor unit is cofibrant. The interest of this axiom comes from the fact that in some HAG contexts of interest the tensor unit is not cofibrant, eg in brave new algebraic geometry. 
Acknowledgements The author is grateful to Fank Neumann and Behrang Noohi for their interest and for conversations related to the contents of this paper. He is also very grateful to an anonymous referee for very useful comments, in particular for pointing out that (1-2) and $\underline{\operatorname{Alg}}_{F}(\phi)$ are flat in derived algebraic geometry.

The author was partially supported by the Andalusian Ministry of Economy, Innovation and Science under the grant FQM-5713, by the Spanish Ministry of Education and Science under the MEC-FEDER grant MTM2010-15831, and by the Government of Catalonia under the grant SGR-119-2009.

\section{Operads and algebras}

This section contains some background about operads and their algebras. All operads considered in this paper are nonsymmetric.

We will be mostly dealing with (symmetric) monoidal model categories. Model structures are not really relevant in this section, but we prefer to fix now the standard assumptions needed in most of the paper.

Definition 2.1 A monoidal model category $\mathscr{C}$ in the sense of Hovey [13, Definition 4.2.6] is a biclosed monoidal category endowed with a model structure such that the pushout product axiom (see Schwede and Shipley [23, Definition 3.1]) and the unit axiom hold. These two axioms imply that the homotopy category Ho $\mathscr{C}$ has an induced biclosed monoidal structure [13, Section 4.3]. The tensor product is denoted by $\otimes$ and the tensor unit by $\mathbb{I}$. If we need to distinguish between different monoidal categories we add a subscript, eg $\otimes_{\mathscr{C}}$ and $\mathbb{I}_{\mathscr{C}}$.

We further assume that monoidal model categories satisfy the monoid axiom [20, Definition 9.1] and the strong unit axiom [21, Definition A.9], recalled in Remark A.2 below. They guarantee the existence of transferred model structures on monoids, operads, algebras over operads etc.; see [23;20]. The strong unit axiom allows the transfer of Quillen equivalences and always holds if the tensor unit is cofibrant; see [21].

We will also assume that all monoidal model categories are cofibrantly generated by sets of generating (trivial) cofibrations with presentable sources.

A symmetric monoidal model category $\mathcal{V}$ is a monoidal model category as above whose underlying monoidal category is symmetric. In this case the monoid axiom simplifies; see [23, Definition 3.3].

For the rest of this section, we fix a symmetric monoidal model category $\mathscr{V}$. 
Definition 2.2 An operad $\mathcal{O}$ in $\mathscr{V}$ is a sequence $\mathcal{O}=\{\mathcal{O}(n)\}_{n \geq 0}$ of objects in $\mathscr{V}$ equipped with an identity,

$$
\operatorname{id}_{\mathcal{O}}: \mathbb{I} \rightarrow \mathcal{O}(1)
$$

and composition laws, $1 \leq i \leq p, q \geq 0$,

$$
\circ_{i}: \mathcal{O}(p) \otimes \mathcal{O}(q) \longrightarrow \mathcal{O}(p+q-1),
$$

satisfying certain associativity and unit equations; see [20, Remark 2.6]. We refer to $\mathcal{O}(n)$ as the arity $n$ component of $\mathcal{O}$.

A morphism of operads $f: \mathcal{O} \rightarrow \mathcal{P}$ is a sequence of morphisms $f(n): \mathcal{O}(n) \rightarrow \mathcal{P}(n)$ in $\mathscr{V}, n \geq 0$, compatible with the identities and composition laws in the obvious way. We denote $\operatorname{Op}(\mathscr{V})$ the model category of operads in $\mathscr{V}$. An operad morphism $f$ is a fibration (resp. weak equivalence) if $f(n)$ is a fibration (resp. weak equivalence) in $\mathscr{V}$ for all $n \geq 0$ [20, Theorem 1.1].

Example 2.3 The unital associative operad uAss is defined as follows: uAss $(n)=\mathbb{I}$ is the tensor unit for all $n \geq 0, \circ_{i}: \operatorname{uAss}(p) \otimes \operatorname{uAss}(q) \rightarrow \operatorname{uAss}(p+q-1)$ is the unit isomorphism $\mathbb{I} \otimes \mathbb{I} \cong \mathbb{I}$ in all cases, and $\mathrm{id}_{\text {uAss }}$ is the identity map in $\mathbb{I}$.

The associative operad Ass is defined by $\operatorname{uAss}(0)=\varnothing$ the initial object and by the existence of an operad morphism $\phi:$ Ass $\rightarrow$ uAss such that $\phi(n)$ is the identity for $n \geq 1$.

Remark 2.4 If $\mathscr{V}=$ Set is the category of sets, the identity is simply an element $\operatorname{id}_{\mathcal{O}} \in \mathcal{O}(1)$ and the associativity and unit equations are:

(1) $\left(x \circ_{i} y\right) \circ_{j} z=\left(x \circ_{j} z\right) \circ_{i+q-1} y$ if $1 \leq j<i$ and $z \in \mathcal{O}(q)$

(2) $\left(x \circ_{i} y\right) \circ_{j} z=x \circ_{i}\left(y \circ_{j-i+1} z\right)$ if $y \in \mathcal{O}(p)$ and $i \leq j<p+i$

(3) $\operatorname{id}_{\mathcal{O}} \circ_{1} x=x$

(4) $x \circ_{i} \operatorname{id}_{\mathcal{O}}=x$

The same happens if $\mathscr{V}=\operatorname{Mod}(\mathbb{k})$ is the category of modules over a commutative ring $\mathbb{k}$.

If $\mathscr{V}=\operatorname{Mod}(\mathbb{k})^{\mathbb{Z}}$ is the category of $\mathbb{Z}$-graded $\mathbb{k}$-modules then the identity must be in degree $0, \operatorname{id}_{\mathcal{O}} \in \mathcal{O}(1)_{0}$, and (1) must be replaced with

$$
\left(x \circ_{i} y\right) \circ_{j} z=(-1)^{|y||z|}\left(x \circ_{j} z\right) \circ_{i+q-1} y \text { if } 1 \leq j<i \text { and } z \in \mathcal{O}(q) .
$$


This reflects the use of the Koszul sign rule in the definition of the symmetry constraint for the tensor product in $\operatorname{Mod}(\mathbb{k})^{\mathbb{Z}}$.

Furthermore, if $\mathscr{V}=\mathrm{Ch}(\mathbb{k})$ is the category of differential graded $\mathbb{k}$-modules, in addition the identity must be a cycle, $d\left(\operatorname{id}_{\mathcal{O}}\right)=0$, and the differential must behave as a derivation with respect to all composition laws,

$$
d\left(x \circ_{i} y\right)=d(x) \circ_{i} y+(-1)^{|x|} x \circ_{i} d(y) .
$$

In this paper, differentials have degree $|d|=-1$, ie we consider chain complexes.

In all these cases, the compatibility conditions an operad morphism $f: \mathcal{O} \rightarrow \mathcal{P}$ must satisfy are

$$
f(p)(x) \circ_{i} f(q)(y)=f(p+q-1)\left(x \circ_{i} y\right), \quad f(1)\left(\operatorname{id}_{\mathcal{O}}\right)=\operatorname{id}_{\mathcal{P}} .
$$

Definition 2.5 A model $\mathscr{V}$-algebra $\mathscr{C}$ is a monoidal model category, in the sense of Definition 2.1, equipped with a left Quillen functor $z: \mathscr{V} \rightarrow \mathscr{C}$ and natural isomorphisms

$$
\text { multiplication: } \begin{aligned}
z(X) \otimes_{\mathscr{C}} z\left(X^{\prime}\right) & \longrightarrow z\left(X \otimes_{\mathscr{V}} X^{\prime}\right), \\
\text { unit: } \mathbb{I}_{\mathscr{C}} & \longrightarrow z\left(\mathbb{I}_{\mathscr{V}}\right), \\
\zeta(X, Y): z(X) \otimes_{\mathscr{C}} Y & \longrightarrow Y \otimes_{\mathscr{C}} z(X),
\end{aligned}
$$

satisfying some coherence laws; see Borceux [6, Definition 6.4.1] and [20, Section 7]. We also assume that $z$ satisfies the $\mathbb{I}$-cofibrant axiom [21, Definition B.6]. Hence, Quillen equivalent $\mathscr{V}$-algebras have Quillen equivalent categories of algebras over a same nice enough operad; see [21, Theorem D.11].

A symmetric model $\mathscr{V}$-algebra $\mathscr{C}$ is a symmetric monoidal model category equipped with a strong symmetric monoidal left Quillen functor $z: \mathscr{V} \rightarrow \mathscr{C}$ satisfying the $\mathbb{I}-$ cofibrant axiom.

In Hovey's terminology, a model $\mathscr{V}$-algebra is the same as a central monoidal $\mathscr{V}$-model category, and a symmetric model $\mathscr{V}$-algebra is a symmetric $\mathscr{V}$-model category; see the paragraph after [13, Definition 4.2.20].

For the rest of this section, let us fix a model $\mathscr{V}$-algebra $\mathscr{C}$.

Example 2.6 The trivial example of symmetric model $\mathscr{V}$-algebra is $\mathscr{b}=\mathscr{V}$ and $z$ the identity functor. 
Remark 2.7 The functor $z(-) \otimes Y: \mathscr{V} \rightarrow \mathscr{C}$ has a right adjoint

$$
\operatorname{Hom}_{\mathscr{C}}(Y,-): \mathscr{b} \longrightarrow \mathscr{V}
$$

for any $Y$ in $\mathscr{b}$. These morphism objects define a tensored $\mathscr{V}$-enrichment of $\mathscr{C}$ over $\mathscr{V}$; see Janelidze and Kelly [14, Appendix]. Moreover, this enrichment can be enhanced to a monoidal $\mathscr{V}$-category structure on $\mathscr{C}$. For this, given two objects $Y$ and $Y^{\prime}$ in $\mathscr{C}$, we define the evaluation morphism

$$
\text { evaluation: } z\left(\operatorname{Hom}_{\mathscr{C}}\left(Y, Y^{\prime}\right)\right) \otimes Y \longrightarrow Y^{\prime}
$$

as the adjoint of the identity in $\operatorname{Hom}_{\mathscr{C}}\left(Y, Y^{\prime}\right)$, and given two other objects $X$ and $X^{\prime}$ in $\mathscr{b}$ we define the morphism in $\mathscr{V}$

$$
\otimes_{\mathscr{C}}: \operatorname{Hom}_{\mathscr{C}}\left(X, X^{\prime}\right) \otimes_{\mathscr{V}} \operatorname{Hom}_{\mathscr{C}}\left(Y, Y^{\prime}\right) \longrightarrow \operatorname{Hom}_{\mathscr{C}}\left(X \otimes_{\mathscr{C}} Y, X^{\prime} \otimes_{\mathscr{C}} Y^{\prime}\right)
$$

as the adjoint of:

$$
\begin{gathered}
z\left(\operatorname{Hom}_{\mathscr{C}}\left(X, X^{\prime}\right) \otimes_{\mathscr{V}} \operatorname{Hom}_{\mathscr{C}}\left(Y, Y^{\prime}\right)\right) \otimes_{\mathscr{C}} X \otimes_{\mathscr{C}} Y \\
\text { multiplication }{ }^{-1} \otimes_{\mathscr{C}} \operatorname{id}_{X} \otimes_{\mathscr{C}} Y \\
z\left(\operatorname{Hom}_{\mathscr{C}}\left(X, X^{\prime}\right)\right) \otimes_{\mathscr{C}} z\left(\operatorname{Hom}_{\mathscr{C}}\left(Y, Y^{\prime}\right)\right) \otimes_{\mathscr{C}} X \otimes_{\mathscr{C}} Y \\
\zeta\left(\operatorname{Hom}_{\mathscr{C}}\left(Y, Y^{\prime}\right), X\right) \mid \cong \\
z\left(\operatorname{Hom}_{\mathscr{C}}\left(X, X^{\prime}\right)\right) \otimes_{\mathscr{C}} X \otimes_{\mathscr{C}} z\left(\operatorname{Hom}_{\mathscr{C}}\left(Y, Y^{\prime}\right)\right) \otimes_{\mathscr{C}} Y \\
\text { evaluation } \otimes_{\mathscr{C}} \text { evaluation } \\
\downarrow \\
X^{\prime} \otimes_{\mathscr{C}} Y^{\prime}
\end{gathered}
$$

Definition 2.8 Let $\mathcal{O}$ be an operad in $\mathscr{V}$. An $\mathcal{O}$-algebra $A$ in $\mathscr{b}$ is an object of $\mathscr{C}$ equipped with structure morphisms

$$
v_{n}: z(\mathcal{O}(n)) \otimes A^{\otimes n} \longrightarrow A, \quad n \geq 0,
$$

which satisfy compatibility relations with the composition laws and the unit of $\mathcal{O}$; see [20, Definition 7.1].

An $\mathcal{O}$-algebra morphism $g: A \rightarrow B$ is a morphism in $\mathscr{C}$ compatible with the structure morphisms in the obvious way; see again [20, Definition 7.1]. We denote $\operatorname{Alg}_{\mathscr{C}}(\mathcal{O})$ the model category of $\mathcal{O}$-algebras in $\mathscr{C}$, where a morphism is a fibration (resp. weak equivalence) if its underlying morphism in $\mathscr{C}$ is a fibration (resp. weak equivalence) [20, Theorem 1.2]. 
Example 2.9 Algebras over the unital associative operad uAss in Example 2.3 are unital associative algebras in $\mathscr{C}$, ie monoids, and algebras over the associative operad Ass are associative algebras in $\mathscr{C}$, ie nonunital monoids.

Remark 2.10 Let $\mathscr{V}=\mathscr{C}$ and $z$ the identity functor. If $\mathscr{V}=$ Set or $\operatorname{Mod}(\mathbb{k})$, as in Remark 2.4, and we denote

$$
v_{n}\left(x, a_{1}, \ldots, a_{n}\right)=x\left(a_{1}, \ldots, a_{n}\right),
$$

then the relations an $\mathcal{O}$-algebra must satisfy are:

(1) If $x \in \mathcal{O}(p)$ and $y \in \mathcal{O}(q)$, then

$$
\begin{aligned}
& \left(x \circ_{i} y\right)\left(a_{1}, \ldots, a_{p+q-1}\right) \\
& \quad=x\left(a_{1}, \ldots, a_{i-1}, y\left(a_{i}, \ldots, a_{i+q-1}\right), a_{i+q}, \ldots, a_{p+q-1}\right)
\end{aligned}
$$

(2) $\operatorname{id}_{\mathcal{O}}(a)=a$

If $\mathscr{V}=\operatorname{Mod}(\mathbb{k})^{\mathbb{Z}}$ then (1) must be replaced with:

$\left(1^{\prime}\right)$ If $x \in \mathcal{O}(p)$ and $y \in \mathcal{O}(q)$, then

$$
\begin{aligned}
& \left(x \circ_{i} y\right)\left(a_{1}, \ldots, a_{p+q-1}\right) \\
& \quad=(-1)^{|y| \sum_{j=1}^{i-1}\left|a_{j}\right|} x\left(a_{1}, \ldots, a_{i-1}, y\left(a_{i}, \ldots, a_{i+q-1}\right), a_{i+q}, \ldots, a_{p+q-1}\right) .
\end{aligned}
$$

Moreover, if $\mathscr{V}=\mathrm{Ch}(\mathbb{k})$, then in addition the following derivation-like formula holds: $d\left(x\left(a_{1}, \ldots, a_{n}\right)\right)=d(x)\left(a_{1}, \ldots, a_{n}\right)+\sum_{i=1}^{n}(-1)^{|x|+\sum_{j=1}^{i-1}\left|a_{j}\right|} x\left(a_{1}, \ldots, d\left(a_{i}\right), \ldots, a_{n}\right)$

In all these cases the compatibility conditions that an $\mathcal{O}$-algebra morphism $g: A \rightarrow B$ must satisfy are

$$
g\left(x\left(a_{1}, \ldots, a_{n}\right)\right)=x\left(g\left(a_{1}\right), \ldots, g\left(a_{n}\right)\right) .
$$

Algebras over an operad can be alternatively described by means of endomorphism operads.

Definition 2.11 The endomorphism operad of an object $Y$ in $\mathscr{C}$ is the operad $\operatorname{End}_{\mathscr{C}}(Y)$ in $\mathscr{V}$ with

$$
\operatorname{End}_{\mathscr{C}}(Y)(n)=\operatorname{Hom}_{\mathscr{C}}\left(Y^{\otimes n}, Y\right) \text {. }
$$

The identity of this operad is the $\mathscr{V}$-enriched identity in $Y$. Composition laws are described in [20, Definition 7.1]. 
Remark 2.12 In the first (resp. last) two examples of Remark 2.10, composition laws in the endomorphism operad are given by equation (1) (resp. $\left(1^{\prime}\right)$ ).

Lemma 2.13 For any operad $\mathcal{O}$ in $\mathscr{V}$ and any object $Y$ in $\mathscr{C}$, there is a bijection between the morphisms $\mathcal{O} \rightarrow \operatorname{End}_{\mathscr{C}}(Y)$ in $\mathrm{Op}(\mathscr{V})$ and the $\mathcal{O}$-algebra structures on $Y$.

Proof The adjoint of the morphism $\mathcal{O}(n) \rightarrow \operatorname{End}_{\mathscr{C}}(Y)(n)=\operatorname{Hom}_{\mathscr{C}}\left(Y^{\otimes n}, Y\right)$ is the structure morphism $v_{n}: z(\mathcal{O}(n)) \otimes Y^{\otimes n} \rightarrow Y$.

Diagrams of algebras over an operad $\mathcal{O}$ can also be descried as $\mathcal{O}$-algebras in a category of diagrams.

Given a small category $I$, the category of $I$-shaped diagrams in $\mathscr{C}$ is the category $\mathscr{C} I$ of functors $I \rightarrow \mathscr{C}$ and natural transformations between them. This category inherits from $\mathscr{C}$ a model $\mathscr{V}$-algebra structure. A morphism $Y \rightarrow Y^{\prime}$ between diagrams $Y, Y^{\prime}: I \rightarrow \mathscr{C}$ is a fibration (resp. weak equivalence) if $Y(i) \rightarrow Y^{\prime}(i)$ is a fibration (resp. weak equivalence) for all objects $i$ in $I$. The tensor product of $Y$ and $Y^{\prime}$ is defined as

$$
\left(Y \otimes Y^{\prime}\right)(i)=Y(i) \otimes Y^{\prime}(i), \quad i \in I,
$$

and the tensor unit of $\mathscr{C}^{I}$ is the constant diagram $\mathbb{I}_{\mathscr{C} I}(i)=\mathbb{I}_{\mathscr{C}}$. The rest of the structure is given by

$$
z^{I}: \mathscr{V} \longrightarrow Z\left(\mathscr{C}^{I}\right), \quad z^{I}(X)(i)=z(X), \quad \zeta^{I}(X, Y)(i)=\zeta(X, Y(i)) .
$$

The right adjoint of $z^{I}(-) \otimes Y: \mathscr{V} \rightarrow \mathscr{C}^{I}$ is the functor

$$
\operatorname{Hom}_{\mathscr{C} I}(Y,-): \mathscr{C}^{I} \longrightarrow \mathscr{V}
$$

defined by the following end in $\mathscr{V}$ (see Mac Lane [18, IX.5]):

$$
\operatorname{Hom}_{\mathscr{C} I}\left(Y, Y^{\prime}\right)=\int_{i \in I} \operatorname{Hom}_{\mathscr{C}}\left(Y(i), Y^{\prime}(i)\right)
$$

The next result follows readily from the previous lemma and the universal property of an end.

Corollary 2.14 For any operad $\mathcal{O}$ in $\mathscr{V}$, any small category $I$, and any diagram $Y: I \rightarrow \mathscr{C}$, there is a bijection between the morphisms $\mathcal{O} \rightarrow \operatorname{End}_{\mathscr{C} I}(Y)$ in $\operatorname{Op}(\mathscr{V})$ and the collections of $\mathcal{O}$-algebra structures on the objects $Y(i)$ in $\mathscr{b}, i \in I$, such that the morphisms in the diagram are $\mathcal{O}$-algebra morphisms. 
Definition 2.15 A $\mathscr{V}$-algebra functor is a lax monoidal functor between $\mathscr{V}$-algebras $F: \mathscr{C} \rightarrow \mathscr{D}$ equipped with a monoidal natural isomorphism $F z_{\mathscr{C}}(X) \cong z_{\mathscr{D}}(X)$ such that the diagram in [13, Definition 4.1.11] commutes (after replacing Hovey's $i$ with our $z$, which is just a matter of notation).

Remark 2.16 A $\mathscr{V}$-algebra functor $F: \mathscr{C} \rightarrow \mathscr{D}$ is $\mathscr{V}$-enriched. For any two objects $Y$ and $Z$ of $\mathscr{C}$, the morphism $F(Y, Z): \operatorname{Hom}_{\mathscr{C}}(Y, Z) \rightarrow \operatorname{Hom}_{\mathscr{D}}(F(Y), F(Z))$ in $\mathscr{V}$ is the adjoint of

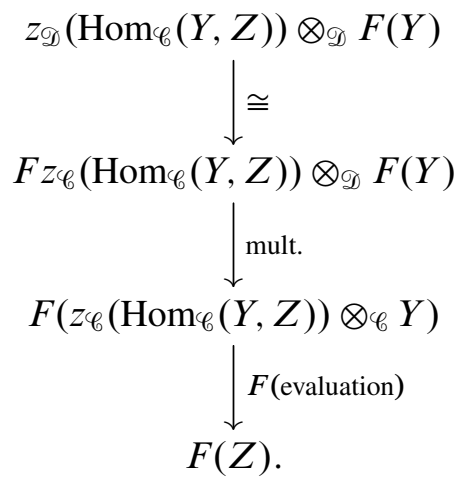

A functor $F: J \rightarrow I$ between small categories induces by precomposition a strong monoidal $\mathscr{V}$-algebra functor

$$
F^{*}: \mathscr{C}^{I} \longrightarrow \mathscr{C}^{J}, \quad F^{*}(Y)=Y F .
$$

The morphisms between morphism objects in $\mathscr{V}$,

$$
F^{*}(Y, Z): \operatorname{Hom}_{\mathscr{C} I}(Y, Z) \longrightarrow \operatorname{Hom}_{\mathscr{C} J}(Y F, Z F),
$$

are defined by the universal property of an end. These morphisms give rise to morphisms in $\mathrm{Op}(\mathscr{V})$ between endomorphism operads,

$$
F^{*}: \operatorname{End}_{\mathscr{C} I}(Y) \longrightarrow \operatorname{End}_{\mathscr{C} J}(Y F) .
$$

Denote $\Delta$ the simplex category, whose objects are the finite ordinals

$$
\boldsymbol{n}=\{0<\cdots<n\}, \quad n \geq 0,
$$

and morphisms are nondecreasing maps. These ordinals are regarded as categories with morphisms going upwards $i \rightarrow j, i \leq j$. The category $\Delta$ is generated by the coface and codegeneracy maps,

$$
d^{i}: \boldsymbol{n}-\mathbf{1} \longrightarrow \boldsymbol{n}, \quad s^{i}: \boldsymbol{n}+\mathbf{1} \longrightarrow \boldsymbol{n}, \quad 0 \leq i \leq n,
$$


which satisfy the duals of the usual simplicial relations. As usual, we denote $\left(d^{i}\right)^{*}=d_{i}$ and $\left(s^{i}\right)^{*}=s_{i}$.

A morphism in $\mathscr{C}$ is the same as a functor $\mathbf{1} \rightarrow \mathscr{C}$. Applying the previous corollary to $I=1$, we deduce the following characterization of $\mathcal{O}$-algebra morphisms.

Corollary 2.17 Given an operad $\mathcal{O}$ in $\mathscr{V}$ and two $\mathcal{O}$-algebras in $\mathscr{C}, X$ and $Y$, defined by morphisms $f_{X}: \mathcal{O} \rightarrow \operatorname{End}_{\mathscr{C}}(X)$ and $f_{Y}: \mathcal{O} \rightarrow \operatorname{End}_{\mathscr{C}}(Y)$ in $\mathrm{Op}(\mathscr{V})$, there is a bijection between the morphisms $g: X \rightarrow Y$ in $\operatorname{Alg}_{\mathscr{C}}(\mathcal{O})$ and the morphisms $g: X \rightarrow Y$ in $\mathscr{C}$ such that there exists a morphism $f_{g}: \mathcal{O} \rightarrow \operatorname{End}_{\mathscr{C}_{1}}(g)$ making the following diagram commutative:

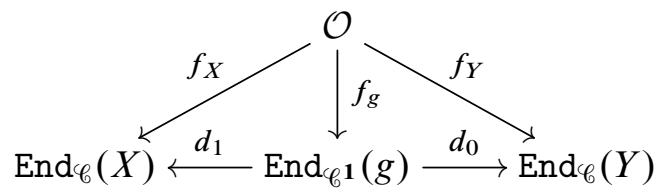

In this corollary, the morphism $f_{g}$ is unique provided it exists since, by definition of end, we have pullback diagrams as follows, $n \geq 0$ :

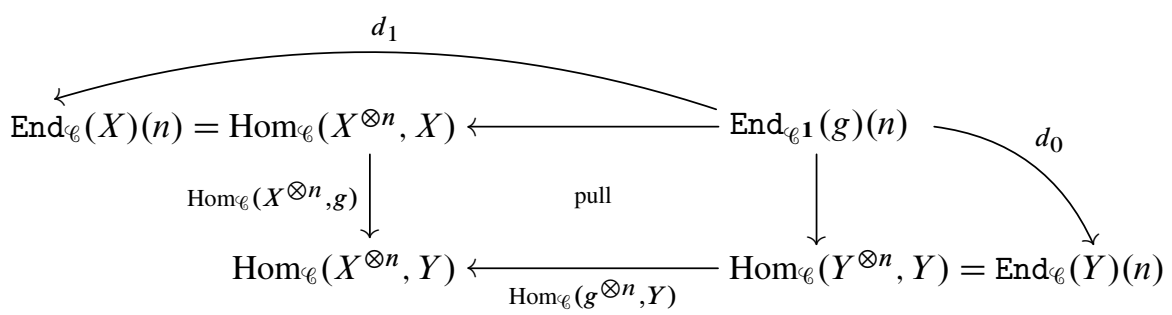

\section{Homotopy invariance of endomorphism operads}

Let $\mathscr{V}$ be a symmetric monoidal model category and $\mathscr{C}$ a model $\mathscr{V}$-algebra. In this section we establish the homotopy invariance properties in $\mathrm{Op}(\mathscr{V})$ of endomorphism operads of objects in $\mathscr{C}$. This is a standard way of transferring algebra structures along weak equivalences in $\mathscr{C}$, compare Berger and Moerdijk [5, Theorem 3.5], and it will have further applications in Section 4.

Lemma 3.1 Given a cofibrant object $Y$ in $\mathscr{C}$, the adjoint pair

$$
\mathscr{V} \underset{\operatorname{Hom}_{\mathscr{C}}(Y,-)}{\stackrel{z(-) \otimes Y}{\rightleftarrows}} \mathscr{C}
$$

is a Quillen pair. 
Proof Since $z$ is a left Quillen functor and $Y$ is cofibrant then $z(-) \otimes Y$ is a left Quillen functor by the pushout product axiom.

Corollary 3.2 If $Y$ is a fibrant-cofibrant object in $\mathscr{b}_{\text {then }} \operatorname{End}_{\mathscr{C}}(Y)$ is a fibrant operad in $\mathrm{Op}(\mathscr{V})$.

Proof The tensor powers $Y^{\otimes n}$ are cofibrant for $n \geq 1$ by the pushout product axiom. Hence, by the previous lemma, the objects $\operatorname{End}_{\mathscr{C}}(Y)(n)=\operatorname{Hom}_{\mathscr{C}}\left(Y^{\otimes n}, Y\right)$ are fibrant in $\mathscr{V}$ since $Y$ is fibrant and $\operatorname{Hom}_{\mathscr{C}}\left(Y^{\otimes n},-\right)$ is a right Quillen functor. Similarly, $\operatorname{End}_{\mathscr{C}}(Y)(0)=\operatorname{Hom}_{\mathscr{C}}(\mathbb{I}, Y)$ is fibrant since $Y$ is fibrant and $\operatorname{Hom}_{\mathscr{C}}(\mathbb{I},-)$ is a right Quillen functor. It is actually the right adjoint of the left Quillen functor $z$.

Lemma 3.3 If $f: X \rightarrow Y$ is a cofibration (resp. trivial cofibration) and $Z$ is a fibrant object in $\mathscr{C}$, then the induced morphism

$$
\operatorname{Hom}_{\mathscr{C}}(f, Z): \operatorname{Hom}_{\mathscr{C}}(Y, Z) \longrightarrow \operatorname{Hom}_{\mathscr{C}}(X, Z)
$$

is a fibration (resp. trivial fibration).

Proof Consider a commutative square of solid arrows in $\mathscr{V}$,

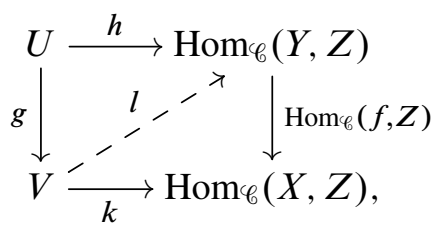

where $g$ is a trivial cofibration (resp. cofibration). We must construct a diagonal morphism $l$ such that the two triangles commute.

The solid diagram (3-1) is the same as a commutative square in $\mathscr{C}$

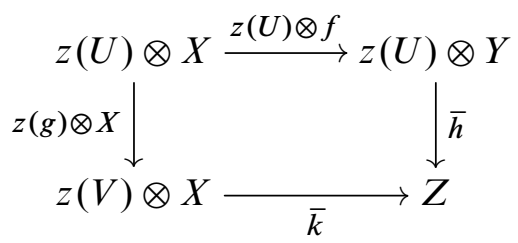

where $\bar{h}$ and $\bar{k}$ are the adjoints of $h$ and $k$, respectively. We will consider the induced morphism from the pushout of the left upper corner to $Z$ : 


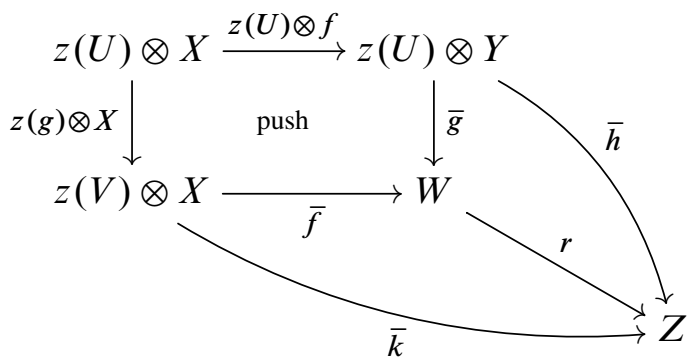

Since $z$ is a left Quillen functor, $z(g)$ is a trivial cofibration (resp. cofibration), hence the pushout product $z(g) \odot f: W \rightarrow z(V) \otimes Y$ of $z(g)$ and $f$, see [20, Section 4], is a trivial cofibration by the pushout product axiom. Since $Z$ is fibrant, there exists a morphism $\bar{l}: z(V) \otimes Y \rightarrow Z$ fitting into a commutative diagram:

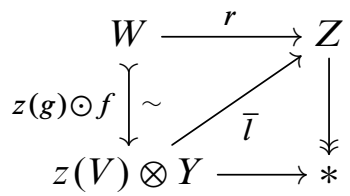

Here $*$ denotes the final object. We can take $l: V \rightarrow \operatorname{Hom}_{\mathscr{C}}(Y, Z)$ to be the adjoint of $\bar{l}$.

The following result is a consequence of the previous lemma and Ken Brown's Lemma.

Corollary 3.4 For any fibrant object $Z$ in $\mathscr{C}$, the functor $\operatorname{Hom}_{\mathscr{C}}(-, Z)$ : $\mathscr{C}^{\text {op }} \rightarrow \mathscr{V}$ takes weak equivalences between cofibrant objects in $\mathscr{b}$ to weak equivalences in $\mathscr{V}$.

An immediate consequence of the following proposition is that the endomorphism operad of a fibrant-cofibrant object in $\mathscr{C}$ is an invariant of its weak homotopy type.

Proposition 3.5 Let $f: X \rightarrow Y$ be a morphism in $\mathscr{C}$. Consider the induced morphisms between endomorphism operads in $\mathrm{Op}(\mathscr{V})$,

$$
\operatorname{End}_{\mathscr{C}}(X) \stackrel{d_{1}}{\longleftarrow} \operatorname{End}_{\mathscr{C} 1}(f) \stackrel{d_{0}}{\longrightarrow} \operatorname{End}_{\mathscr{C}}(Y) .
$$

(1) If $f$ is a (trivial) fibration and $X$ is cofibrant then $d_{0}$ is a (trivial) fibration.

(2) If $f$ is a trivial fibration between fibrant-cofibrant objects then $d_{1}$ is a weak equivalence.

(3) If $f$ is a (trivial) cofibration between cofibrant objects and $Y$ is fibrant then $d_{1}$ is a (trivial) fibration.

(4) If $f$ is a trivial cofibration between fibrant-cofibrant objects then $d_{0}$ is a weak equivalence. 
Proof Consider the pullback diagram (2-2). By the pushout product axiom, the tensor powers $X^{\otimes n}$ are cofibrant in the four cases. Hence, under the assumptions of (1) and (2), $\operatorname{Hom}_{\mathscr{C}}\left(X^{\otimes n}, f\right)$ is a (trivial) fibration by Lemma 3.1. Now (1) follows from the fact that (trivial) fibrations are closed under pullbacks.

Under the hypotheses of (2), the pushout product axiom and Ken Brown's Lemma show that the tensor powers $f^{\otimes n}$ are weak equivalences between cofibrant objects, hence $\operatorname{Hom}_{\mathscr{C}}\left(f^{\otimes n}, Y\right)$ is a weak equivalence between fibrant objects by Lemma 3.3 and Corollary 3.4. Therefore (2) follows, since the pullback of a weak equivalence between fibrant objects along a fibration is a weak equivalence.

Under the assumptions of (3) and (4), the tensor powers $f^{\otimes n}$ are (trivial) cofibrations between cofibrant objects by the pushout product axiom. Hence, $\operatorname{Hom}_{\mathscr{C}}\left(f^{\otimes n}, Y\right)$ is a (trivial) fibration between fibrant objects by Lemma 3.3. Notice that (3) follows from the same reason as (1). Moreover, under the hypotheses of (4), $\operatorname{Hom}_{\mathscr{C}}\left(X^{\otimes n}, f\right)$ is a weak equivalence between fibrant objects by Lemma 3.1 and Ken Brown's Lemma, hence (4) follows from the same reason as (2).

The following result follows from the usual lifting properties, Proposition 3.5 and Corollary 2.17.

Corollary 3.6 Let $\mathcal{O}$ be a cofibrant operad in $\mathscr{V}$ and $f: X \stackrel{\sim}{\rightarrow} Y$ a weak equivalence in $\mathscr{b}$.

(1) If $f$ is a trivial fibration, $X$ is cofibrant, and $Y$ is an $\mathcal{O}$-algebra, then there exists an $\mathcal{O}$-algebra structure on $X$ such that $f$ becomes a morphism of $\mathcal{O}$-algebras.

(2) If $f$ is a trivial cofibration, $X$ is cofibrant, $Y$ is fibrant, and $X$ is an $\mathcal{O}$-algebra, then there exists an $\mathcal{O}$-algebra structure on $Y$ such that $f$ becomes a morphism of $\mathcal{O}$-algebras.

The following corollary of Proposition 3.5 will be very useful in our study of spaces of algebras.

Corollary 3.7 Let $f_{i}: X_{i} \rightarrow X_{i+1}$ be trivial fibrations between fibrant-cofibrant objects in $\mathscr{C}, 0 \leq i \leq n$. The induced morphisms between endomorphism operads in $\mathrm{Op}(\mathscr{V})$,

$\operatorname{End}_{\mathscr{C}} n\left(X_{0} \rightarrow \cdots \rightarrow X_{n}\right) \stackrel{d_{n+1}}{\longleftarrow} \operatorname{End}_{\mathscr{C} n+1}\left(X_{0} \rightarrow \cdots \rightarrow X_{n} \rightarrow X_{n+1}\right) \stackrel{d_{0}^{n+1}}{\longrightarrow} \operatorname{End}_{\mathscr{C}}\left(X_{n+1}\right)$,

are a weak equivalence and a trivial fibration, respectively. Moreover, these three operads are fibrant in $\mathrm{Op}(\mathscr{V})$. 
Proof By induction on $n$. The case $n=0$ follows directly from Proposition 3.5 and Corollary 3.2. Assume $n>0$. Consider the following pull back diagram:

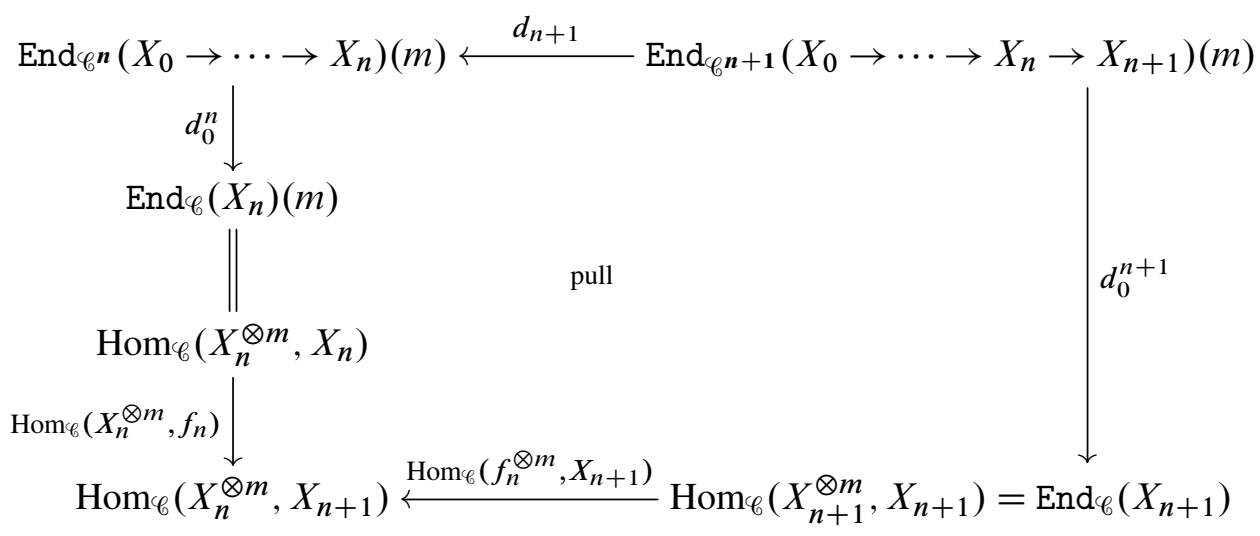

The morphism $d_{0}^{n}$ is a trivial fibration by induction hypothesis. We showed within the proof of Proposition 3.5 that, under the hypotheses of this corollary, the morphisms $\operatorname{Hom}_{\mathscr{C}}\left(X_{n}^{\otimes m}, f_{n}\right)$ and $\operatorname{Hom}_{\mathscr{C}}\left(f_{n}^{\otimes m}, X_{n+1}\right)$ are a trivial fibration and a weak equivalence between fibrant objects, respectively. Hence, the first part of the statement follows from the facts that trivial fibrations are closed under composition and pullbacks and that weak equivalences between fibrant objects are closed under pullbacks along fibrations. Finally, the first and last operads are fibrant by induction hypothesis, and the middle one is fibrant since $d_{0}^{n+1}$ is a trivial fibration with fibrant target.

\section{Spaces of algebras}

The main result of this section (Theorem 4.6) generalizes Rezk's [22, Theorem 1.1.5] in the nonsymmetric context.

We will be concerned with the classifying space $|w M|$ (also called nerve) of the subcategory of weak equivalences $w \mathcal{M}$ in a model category $\mathcal{M}$, and more generally in appropriate subcategories of a model category. The classifying space $|w M|$ is sometimes referred to as the classification complex or space of $\mathcal{M}$, compare Dwyer and Kan [10]. This need not be an honest simplicial set because $M$ may have a proper class of objects. Moreover, it need not be homotopically small in the sense of Dwyer and Kan [9]. We will overlook this fact since there are well known ways of patching these problems: restricting to the closure of a set of objects under weak equivalences, working with Grothendieck universes etc.

Denote $\mu_{c}$ and $\mu_{f}$ the full subcategories of cofibrant and fibrant objects in $\mathcal{M}$, respectively. The inclusions induce weak equivalences $\left|w M_{c}\right| \simeq|w \mathcal{M}| \simeq\left|w M_{f}\right|$ 
(see [22, Lemma 4.2.4].) Moreover, a Quillen equivalence $F$ : $\mathcal{M} \rightleftarrows \mathcal{N}$ : $G$ induces weak equivalences $\left|w \mathcal{M}_{c}\right| \simeq\left|w \mathcal{N}_{c}\right|$ and $\left|w \mathcal{N}_{f}\right| \simeq\left|w \mathcal{M}_{f}\right|$ by Cisinski [7, Proposition 2.3, Exemple 2.5 and Théorème 2.9]. Therefore, if in addition $G$ or $F$ preserves weak equivalences then it induces a weak equivalence $|w M| \simeq|w \mathcal{N}|$.

In this paper, the word space is a synonym of simplicial set. Bisimplicial sets will be regarded as spaces via the diagonal construction.

Let $\mathscr{V}$ be a symmetric monoidal model category and $\mathscr{C}$ a model $\mathscr{V}$-algebra. A morphism $f: \mathcal{O} \rightarrow \mathcal{P}$ in $\mathrm{Op}(\mathscr{V})$ induces a Quillen pair of change of operad functors,

$$
\operatorname{Alg}_{\mathscr{C}}(\mathcal{O}) \underset{f^{*}}{\stackrel{f_{*}}{\rightleftarrows}} \operatorname{Alg}_{\mathscr{C}}(\mathcal{P})
$$

The functor $f^{*}$ restricts the action of $\mathcal{P}$ to $\mathcal{O}$ along $f$ and is the identity on underlying objects in $\mathscr{C}$, hence it preserves fibrations and weak equivalences. The functor $f_{*}$ is left adjoint to $f^{*}$. This Quillen pair is a Quillen equivalence if $f$ is a weak equivalence and the operads $\mathcal{O}$ and $\mathcal{P}$ are admissible in the sense of the following definition; see [21, Theorem D.4].

Definition 4.1 An object $X$ in $\mathscr{V}$ is $\mathbb{I}$-cofibrant if there exists a cofibration $\mathbb{I} \succ X$ from the tensor unit $\mathbb{I}$. An operad $\mathcal{O}$ in $\mathscr{V}$ is admissible if each $\mathcal{O}(n)$ is cofibrant or I-cofibrant, $n \geq 0$.

Remark 4.2 If $\mathcal{O}$ is a cofibrant operad then $\mathcal{O}(n)$ is cofibrant for $n \neq 1$ and $\mathbb{I}-$ cofibrant for $n=1$ [21, Corollary C.3], hence cofibrant operads are admissible. The unital associative operad in Example 2.3 is $\mathbb{I}$-cofibrant in all arities, and the associative operad is $\mathbb{I}$-cofibrant in positive arities and cofibrant in arity 0 , so they are also admissible despite not being cofibrant.

If $\mathcal{O}^{\bullet}$ is a cosimplicial operad then the contravariant change of base operad functors, like $f^{*}$ in (4-1), induced by cofaces and codegeneracies gives rise to a simplicial category $\operatorname{Alg}_{\mathscr{C}}\left(\mathcal{O}^{\bullet}\right)$.

Lemma 4.3 If $\mathcal{O}^{\bullet}$ is a cosimplicial resolution in the sense of [9, Section 4.3] of an admissible operad $\mathcal{O}$ in $\mathscr{V}$, then there is a weak equivalence

$$
\left|w \operatorname{Alg}_{\mathscr{C}}(\mathcal{O})\right| \stackrel{\sim}{\longrightarrow}\left|w \operatorname{Alg}_{\mathscr{C}}\left(\mathcal{O}^{\bullet}\right)\right| .
$$

Proof The operads $\mathcal{O}^{n}$ are cofibrant, hence admissible, $n \geq 0$, so faces and degeneracies in $\operatorname{Alg}_{\mathscr{C}}\left(\mathcal{O}^{\bullet}\right)$ are right adjoints of a Quillen equivalence, therefore they induce 
weak equivalences on nerves. This implies that the iterated degeneracies induce a weak equivalence

$$
\left|w \operatorname{Alg}_{\mathscr{C}}\left(\mathcal{O}^{0}\right)\right| \stackrel{\sim}{\longrightarrow}\left|w \operatorname{Alg}_{\mathscr{C}}\left(\mathcal{O}^{\bullet}\right)\right| .
$$

For the same reason, the weak equivalence $\mathcal{O}^{0} \stackrel{\sim}{\rightarrow} \mathcal{O}$ induces a weak equivalence

$$
\left|w \operatorname{Alg}_{\mathscr{C}}(\mathcal{O})\right| \stackrel{\sim}{\longrightarrow}\left|w \operatorname{Alg}_{\mathscr{C}}\left(\mathcal{O}^{0}\right)\right| \text {. }
$$

Denote $f w \mathscr{b}_{f c}$ the category of fibrant-cofibrant objects in $\mathscr{C}$ and trivial fibrations between them. Under the assumptions of the previous lemma, define the simplicial category $\mathscr{D}_{\bullet}$ as the following pullback:

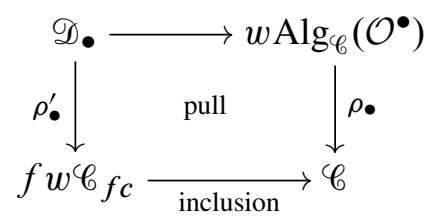

Here we regard the two categories in the bottom as constant simplicial categories, and $\rho_{\bullet}$ is the forgetful simplicial functor. Notice that $\mathscr{D}_{n}$ is the category of trivial fibrations between $\mathcal{O}^{n}$-algebras whose underlying objects in $\mathscr{C}$ are fibrant and cofibrant.

Lemma 4.4 In the situation of the previous paragraph, the horizontal (simplicial) functors in (4-2) induce weak equivalences

$$
\left|f w_{\mathscr{C}_{f c}}\right| \stackrel{\sim}{\longrightarrow}|w \mathscr{C}|, \quad\left|\mathscr{D}_{\bullet}\right| \stackrel{\sim}{\longrightarrow}\left|w \operatorname{Alg}_{\mathscr{C}}\left(\mathcal{O}^{\bullet}\right)\right| .
$$

Proof The first part of the statement follows from [22, Lemmas 4.2.4 and 4.2.5]. Moreover, by the same results together with [21, Corollaries D.2 and D.3], the inclusion $\mathscr{D}_{n} \subset w \operatorname{Alg}_{\mathscr{C}}\left(\mathcal{O}^{n}\right)$ induces a weak equivalence

$$
\left|\mathscr{D}_{n}\right| \stackrel{\sim}{\longrightarrow}\left|w \operatorname{Alg}_{\mathscr{C}}\left(\mathcal{O}^{n}\right)\right|, \quad n \geq 0,
$$

hence the second map in the statement is also a weak equivalence.

Recall that the category of simplices $\Delta K$ of a simplicial set $K$ is the category whose objects are pairs $(\boldsymbol{n}, x)$ with $n \geq 0$ and $x \in K_{n}$. A morphism $\sigma:(\boldsymbol{n}, x) \rightarrow(\boldsymbol{m}, y)$ in $\Delta K$ is a morphism $\sigma: \boldsymbol{n} \rightarrow \boldsymbol{m}$ in $\Delta$ such that the induced map $\sigma^{*}: K_{m} \rightarrow K_{n}$ takes $y$ to $x, \sigma^{*}(y)=x$. This category comes equipped with a natural projection functor $p_{K}: \Delta K \rightarrow \Delta, p_{K}(\boldsymbol{n}, x)=\boldsymbol{n}$. This construction defines a functor from the category of simplicial sets to the category of small categories over $\Delta$,

$$
\begin{gathered}
\mathrm{Set}^{\Delta^{\mathrm{op}}} \longrightarrow \mathrm{Cat} \downarrow \Delta, \\
K \mapsto p_{K} .
\end{gathered}
$$


Lemma 4.5 If $Y$ is a fibrant-cofibrant object in $\mathscr{C}$ and $\mathcal{O}^{\bullet}$ is a cosimplicial resolution in $\mathrm{Op}(\mathscr{V})$, then, with the notation in (4-2), $\left|\rho_{\bullet}^{\prime} \downarrow Y\right|$ is weakly equivalent to $\operatorname{Op}(\mathscr{V})\left(\mathcal{O}^{\bullet}, \operatorname{End}_{\mathscr{C}}(Y)\right)=\operatorname{Map}_{\mathrm{Op}(\mathscr{V})}\left(\mathcal{O}, \operatorname{End}_{\mathscr{C}}(Y)\right)$.

Proof We will use an alternative construction of the bisimplicial set $\left|\rho_{\bullet}^{\prime} \downarrow Y\right|$ in terms of endomorphism operads. By Corollary 2.14,

$$
\left|\rho_{t}^{\prime} \downarrow Y\right|_{s}=\coprod_{X_{0} \rightarrow \cdots \rightarrow X_{s} \rightarrow Y \text { in } f w_{\mathscr{C}_{f c}}} \operatorname{Op}(\mathscr{V})\left(\mathcal{O}^{t}, \operatorname{End}_{\mathscr{C}^{s}}\left(X_{0} \rightarrow \cdots \rightarrow X_{s}\right)\right) .
$$

Notice that the set indexing this coproduct is $\left|\left(f w^{\mathscr{C}_{f}}\right) \downarrow Y\right|_{s}$. In order to describe the bisimplicial structure of $\left|\rho_{\bullet}^{\prime} \downarrow Y\right|$ in terms of the right hand side of (4-3), we consider the functor

$$
\begin{aligned}
E:(\Delta|\mathscr{C}|)^{\mathrm{op}} & \longrightarrow \mathrm{Op}(\mathscr{V}), \\
\left(\boldsymbol{n}, X_{0} \rightarrow \cdots \rightarrow X_{n}\right) & \mapsto \operatorname{End}_{\mathscr{C}}\left(X_{0} \rightarrow \cdots \rightarrow X_{n}\right) .
\end{aligned}
$$

Note that $X_{0} \rightarrow \cdots \rightarrow X_{n}$ is a functor $X: \boldsymbol{n} \rightarrow \mathscr{C}$. Given a morphism $\sigma:(\boldsymbol{n}, X) \rightarrow\left(\boldsymbol{m}, X^{\prime}\right)$ in the category of simplices, then we have that the induced morphism $E(\sigma)$ is $\sigma^{*}: \operatorname{End}_{\mathscr{C}} m\left(X^{\prime}\right) \rightarrow \operatorname{End}_{\mathscr{C}} n(X)$; here we use the notation in (2-1). We also consider the functor

$$
\begin{aligned}
F_{Y}:\left(f w^{\mathscr{C}}{ }_{f c}\right) \downarrow Y & \longrightarrow \mathscr{C}, \\
(X \rightarrow Y) & \mapsto X,
\end{aligned}
$$

and the composite functor

$$
\left(\Delta\left|\left(f w_{\mathscr{C}_{c}}\right) \downarrow Y\right|\right)^{\mathrm{op}} \stackrel{\left(\Delta\left|F_{Y}\right|\right)^{\mathrm{op}}}{\longrightarrow}(\Delta|\mathscr{C}|)^{\mathrm{op}} \stackrel{E}{\longrightarrow} \mathrm{Op}(\mathscr{V}) \stackrel{\mathrm{Op}(\mathscr{V})\left(\mathcal{O}^{\bullet},-\right)}{\longrightarrow} \operatorname{Set}^{\Delta^{\mathrm{op}}} .
$$

Taking the left Kan extension [18, X.3] of this functor along the opposite of the natural projection from the category of simplices to $\Delta$, we obtain a bisimplicial set

$$
\operatorname{Lan}_{p_{\mid\left(f w^{\ell} f_{c} \mid\right) \downarrow Y}^{\text {op }}} \operatorname{Op}(\mathscr{V})\left(\mathcal{O}^{\bullet}, E\left(\Delta\left|F_{Y}\right|\right)^{\mathrm{op}}\right) .
$$

One can easily check that the $(s, t)$ set of this bisimplicial set is the right hand side of (4-3). Moreover, this defines an isomorphism between this bisimplicial set and $\left|\rho_{\bullet}^{\prime} \downarrow Y\right|$.

We need two more functors

$$
\begin{gathered}
L_{Y}, C_{Y}: \Delta\left|\left(f w \mathscr{C}_{f c}\right) \downarrow Y\right| \rightarrow \Delta|\mathscr{b}|, \\
L_{Y}\left(\boldsymbol{n}, X_{0} \rightarrow \cdots \rightarrow X_{n} \rightarrow Y\right)=\left(\boldsymbol{n}+\mathbf{1}, X_{0} \rightarrow \cdots \rightarrow X_{n} \rightarrow Y\right), \\
L_{Y}\left(d^{i}\right)=d^{i}, \quad L_{Y}\left(s^{i}\right)=s^{i}, \\
C_{Y}\left(\boldsymbol{n}, X_{0} \rightarrow \cdots \rightarrow X_{n} \rightarrow Y\right)=(\mathbf{0}, Y),
\end{gathered}
$$


where $C_{Y}$ is constant, and two natural transformations,

$$
\begin{aligned}
\delta_{Y}: \Delta\left|F_{Y}\right| & \Longrightarrow L_{Y}, \quad \delta_{Y}\left(\boldsymbol{n}, X_{0} \rightarrow \cdots \rightarrow X_{n} \rightarrow Y\right)=d^{n+1}, \\
\varsigma_{Y}: C_{Y} & \Longrightarrow L_{Y}, \quad \varsigma_{Y}\left(\boldsymbol{n}, X_{0} \rightarrow \cdots \rightarrow X_{n} \rightarrow Y\right)=\left(d^{0}\right)^{n+1} .
\end{aligned}
$$

For simplicity, denote $K=\left|\left(f w^{C} \mathscr{C}_{f c}\right) \downarrow Y\right|$. We claim that the following morphisms of bisimplicial sets are weak equivalences:

$$
\begin{aligned}
\operatorname{Lan}_{p_{K}^{\text {op }}} \operatorname{Op}(\mathscr{V})\left(\mathcal{O}^{\bullet}, E\left(\Delta\left|F_{Y}\right|\right)^{\mathrm{op}}\right) & \\
& \operatorname{Lan}_{p_{K}^{\text {op }}} \operatorname{Op}(\mathscr{V})\left(\mathcal{O}^{\bullet}, E\left(\delta_{Y}\right)\right) \\
\operatorname{Lan}_{p_{K}^{\text {op }}} \operatorname{Op}(\mathscr{V})\left(\mathcal{O}^{\bullet}, E L_{Y}^{\mathrm{op}}\right) & \\
& \operatorname{Lan}_{p_{K}^{\text {op }}} \operatorname{Op}(\mathscr{V})\left(\mathcal{O}^{\bullet}, E\left(\varsigma_{Y}\right)\right) \\
\operatorname{Lan}_{p_{K}^{\text {op }}} & \operatorname{Op}(\mathscr{V})\left(\mathcal{O}^{\bullet}, E C_{Y}^{\mathrm{op}}\right)
\end{aligned}
$$

It is enough to notice that, at the $(n, \bullet)$ simplicial set, we have the coproduct indexed by $K_{n}$ of the morphisms of simplicial sets obtained by applying $\operatorname{Op}(\mathscr{V})\left(\mathcal{O}^{\bullet},-\right)$ to the weak equivalences between fibrant operads in Corollary 3.7, $n \geq 0$ :

$$
\begin{aligned}
& \bigsqcup_{X_{0} \rightarrow \cdots \rightarrow X_{n} \rightarrow Y \text { in } f w_{\mathscr{C}_{f c}}} \operatorname{Op}(\mathscr{V})\left(\mathcal{O}^{\bullet}, \operatorname{End}_{\mathscr{C} n}\left(X_{0} \rightarrow \cdots \rightarrow X_{n}\right)\right) \\
& \left(d_{n+1}\right)_{*} \uparrow \sim \\
& \bigsqcup_{X_{0} \rightarrow \cdots \rightarrow X_{n} \rightarrow Y \text { in } f w_{\mathscr{C}_{f c}}} \operatorname{Op}(\mathscr{V})\left(\mathcal{O}^{\bullet}, \operatorname{End}_{\mathscr{C} n+1}\left(X_{0} \rightarrow \cdots \rightarrow X_{n} \rightarrow Y\right)\right) \\
& \left(d_{0}^{n+1}\right)_{*} \downarrow \sim \\
& \bigsqcup_{X_{0} \rightarrow \cdots \rightarrow X_{n} \rightarrow Y \text { in } f w_{\mathscr{C}_{f c}}} \mathrm{Op}(\mathscr{V})\left(\mathcal{O}^{\bullet}, \operatorname{End}_{\mathscr{C}}(Y)\right)
\end{aligned}
$$

The functor $\operatorname{Op}(\mathscr{V})\left(\mathcal{O}^{\bullet},-\right)$ takes the weak equivalences between fibrant operads in Corollary 3.7 to weak equivalences of simplicial sets by [9, Corollary 6.4].

In order to complete the definition of the weak equivalence claimed in the statement we notice that

$$
\operatorname{Lan}_{p_{K}^{\text {op }}} \operatorname{Op}(\mathscr{V})\left(\mathcal{O}^{\bullet}, E C_{Y}^{\mathrm{op}}\right)=\left|\left(f w^{\bullet} \mathscr{C}_{f c}\right) \downarrow Y\right| \times \operatorname{Op}(\mathscr{V})\left(\mathcal{O}^{\bullet}, \operatorname{End}_{\mathscr{C}}(Y)\right) .
$$

The category $\left(f w^{C_{f c}}\right) \downarrow Y$ has a final object, the identity in $Y$, hence its nerve is contractible and the projection onto the second factor of the product is a weak equivalence. 
We can now proceed with the main theorem of this section.

Theorem 4.6 Let $\mathcal{O}$ be an admissible operad in $\mathscr{V}$. The homotopy fiber of the map

$$
\left|w \operatorname{Alg}_{\mathscr{C}}(\mathcal{O})\right| \longrightarrow|w \mathscr{C}|
$$

induced by the forgetful functor $\operatorname{Alg}_{\mathscr{b}}(\mathcal{O}) \rightarrow \mathscr{C}$ at a fibrant-cofibrant object $Y$ is $\operatorname{Map}_{\mathrm{Op}(V)}\left(\mathcal{O}, \operatorname{End}_{\mathscr{C}}(Y)\right)$.

Proof We want to apply [22, Lemma 4.2.2] to the simplicial functor $\rho_{\bullet}^{\prime}: \mathscr{D}_{\bullet} \rightarrow f w \mathscr{C}_{f c}$ in (4-2). This lemma of Rezk says that the commutative diagram of simplicial sets

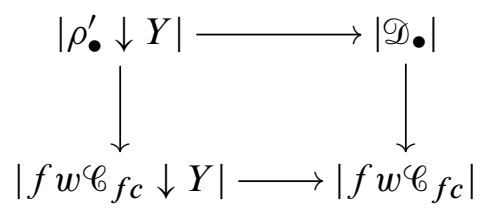

is a homotopy pullback if the maps $\left|\rho_{\bullet}^{\prime} \downarrow Y\right| \rightarrow\left|\rho_{\bullet}^{\prime} \downarrow Y^{\prime}\right|$ induced by maps $g: Y \rightarrow Y^{\prime}$ in $f w^{C} \mathscr{C}_{f c}$ are weak equivalences. In the previous square, the vertical maps are induced by $\rho_{\bullet}^{\prime}$ and the horizontal maps are defined by the source functors on comma categories.

Using Lemma 4.5, we can identify the map $\left|\rho_{\bullet}^{\prime} \downarrow Y\right| \rightarrow\left|\rho_{\bullet}^{\prime} \downarrow Y^{\prime}\right|$ in the homotopy category of spaces with the map represented by the zigzag

$$
\operatorname{Map}_{\mathrm{Op}(\mathscr{V})}\left(\mathcal{O}, \operatorname{End}_{\mathscr{C}}(Y)\right) \longleftarrow \operatorname{Map}_{\mathrm{Op}(\mathscr{V})}\left(\mathcal{O}, \operatorname{End}_{\mathscr{C}} \mathbf{1}(g)\right) \longrightarrow \operatorname{Map}_{\mathrm{Op}(\mathscr{V})}\left(\mathcal{O}, \operatorname{End}_{\mathscr{C}}\left(Y^{\prime}\right)\right)
$$

obtained by applying $\operatorname{Map}_{\mathrm{Op}(\mathscr{V})}(\mathcal{O},-)$ to $d_{1}$ and $d_{0}$ in Proposition 3.5. Since $g$ is a trivial fibration between fibrant-cofibrant objects, $d_{1}$ and $d_{0}$ are weak equivalences between fibrant operads by Corollary 3.2 and Proposition 3.5(1) and (2). This and [9, Corollary 6.4] imply that the previous zigzag consists of two weak equivalences of spaces. Therefore $\left|\rho_{\bullet}^{\prime} \downarrow Y\right| \rightarrow\left|\rho_{\bullet}^{\prime} \downarrow Y^{\prime}\right|$ must be weak equivalence, as we wanted to show.

We have now established that the commutative square in the first paragraph is a homotopy pullback. The space $\left|f w^{\mathscr{C}_{f c}} \downarrow Y\right|$ is weakly contractible since $f w^{\mathscr{C}_{f c}} \downarrow Y$ has a final object (the identity in $Y$ ), hence the homotopy fiber of $\left|\rho_{\bullet}^{\prime}\right|:\left|\mathscr{D}_{\bullet}\right| \rightarrow\left|f w^{\mathscr{C}_{f c}}\right|$ at $Y$ is $\left|\rho_{\bullet}^{\prime} \downarrow Y\right|$, which is weakly equivalent to $\operatorname{Map}_{\mathrm{Op}(\mathscr{V})}\left(\mathcal{O}, \operatorname{End}_{\mathscr{C}}(Y)\right)$ by Lemma 4.5. The map in the statement is weakly equivalent to $\left|\rho_{\bullet}^{\prime}\right|:\left|\mathscr{D}_{\bullet}\right| \rightarrow\left|f w_{\mathscr{C}_{c}}\right|$ by Lemma 4.4. This observation concludes the proof.

Mapping spaces take homotopy colimits in the first variable to homotopy limits. Hence, we deduce the following result. 
Corollary 4.7 The contravariant functor $\mathcal{O} \mapsto\left|w \operatorname{Alg}_{\mathscr{C}}(\mathcal{O})\right|$ defined by the contravariant change of base operad functors, like $f^{*}$ in (4-1), takes homotopy colimits of admissible operads to homotopy limits of spaces.

\section{Stacks of algebras}

In this section we place ourselves in a homotopical algebraic geometry (HAG) context $\left(\mathscr{V}, \mathscr{V}_{0}, \mathscr{A}, \tau, \boldsymbol{P}\right)$ in the sense of $[26,1.3 .2 .13]$. This consists, first of all, of an underlying combinatorial symmetric monoidal model category $\mathscr{V}$, in the sense of [13, Definition 4.2.6], and two full subcategories $\mathscr{V}_{0}, \mathscr{A} \subset \mathscr{V}$ which intuitively play the role of the aisle and the heart of a $t$-structure in a triangulated category, respectively. Among the various required assumptions, the category $\operatorname{Comm}(\mathscr{V})$ of commutative algebras (ie monoids) in $\mathscr{V}$ must carry a model structure with fibrations and weak equivalences defined by the underlying morphisms in $\mathscr{V}$. The model category $\operatorname{Aff}_{\mathscr{V}}=\operatorname{Comm}(\mathscr{V})^{\mathrm{op}}$ of affine stacks is the opposite model category, $\tau$ is a model pretopology on $\mathrm{Aff}_{\mathscr{V}}$, which induces a Grothendieck topology on $\operatorname{Ho}\left(\mathrm{Aff}_{\mathscr{V}}\right)$, and $\boldsymbol{P}$ is a class of morphisms in $\mathrm{Aff}_{\mathscr{V}}$, which are regarded as good enough to define quotients.

Apart from the axioms imposed in [13, Definition 4.2.6] to symmetric monoidal model categories, $\mathscr{V}$ satisfies the strong unit axiom by [26, Assumption 1.1.0.3] and [21, Lemma A.11]. The monoid axiom is not explicitly required in [26], but it is satisfied by all known examples. We will also assume that $\mathscr{V}$ satisfies the monoid axiom, so it is a symmetric monoidal model category in the sense of Definition 2.1.

A simplicial presheaf $F$ is a (contravariant) functor from affine stacks to simplicial sets,

$$
F: \mathrm{Aff}_{\mathscr{V}}^{\mathrm{op}} \longrightarrow \mathrm{Set}^{\Delta^{\mathrm{op}}},
$$

that is, a (covariant) functor from $\operatorname{Comm}(\mathscr{V})$. The category $\operatorname{SPr}(\operatorname{Aff} \mathscr{V})$ of simplicial presheaves carries a model structure where weak equivalences (resp. fibrations) are pointwise weak equivalences (resp. fibrations) of simplicial sets. In particular, a simplicial presheaf is fibrant if and only if its values are Kan complexes.

A stack is a simplicial presheaf $F$ which preserves weak equivalences, finite homotopy products, and satisfies the following descent condition [26, Corollary 1.3.2.4]: given a commutative algebra $A$, a cosimplicial commutative algebra $B^{\bullet}$, and a cosimplicial map $A \rightarrow B^{\bullet}$ corresponding to a $\tau$-hypercover in Aff $_{\mathscr{V}}$, where $A$ is regarded as a constant cosimplicial object, the induced morphism

$$
F(A) \longrightarrow \operatorname{holim}_{n \in \Delta} F\left(B^{n}\right)
$$


is a weak equivalence of spaces. The simplicial presheaf represented by a commutative algebra $A$,

$$
\underline{\mathbb{S p e c}}(A)=\operatorname{Map}_{\operatorname{Comm}(\mathscr{V})}(A,-),
$$

is a stack, that we call affine stack. It is defined by a cosimplicial resolution of $A$ and a functorial fibrant resolution in $\operatorname{Comm}(\mathscr{V})$. In order to turn $\mathbb{R S p e c}$ into a Yoneda-like functor

$$
\mathbb{R} \text { Spec: } \operatorname{Aff}_{\mathscr{V}} \longrightarrow \operatorname{SPr}\left(\operatorname{Aff}_{\mathscr{V}}\right)
$$

we choose a functorial cosimplicial resolution in $\operatorname{Comm}(\mathscr{V})$.

The model category of stacks $\mathrm{Aff}_{\mathscr{V}}^{\sim, \tau}$ is a left Bousfield localization of $\operatorname{SPr}(\operatorname{Aff} \mathscr{V})$ whose fibrant objects are the stacks taking values in Kan complexes. The homotopy category of stacks $\operatorname{St}(\mathscr{V}, \tau)=\operatorname{Ho}\left(\mathrm{Aff}_{\mathscr{V}}^{\sim}, \tau\right)$ can be identified with the full subcategory of $\operatorname{Ho}\left(\operatorname{SPr}\left(\mathrm{Aff}_{\mathscr{V}}\right)\right)$ spanned by stacks. The homotopy limit of a diagram of stacks is the same in $\operatorname{Aff}_{\mathscr{V}}^{\sim}, \tau$ and in $\operatorname{SPr}\left(\mathrm{Aff}_{\mathscr{V}}\right)$, ie it can be computed as a pointwise homotopy limit in the category of spaces. A simplicial presheaf weakly equivalent to a stack in $\operatorname{SPr}\left(\mathrm{Aff}_{\mathscr{V}}\right)$ is itself a stack. A map between stacks is a weak equivalence in $\mathrm{Aff}_{\mathscr{V}}^{\sim, \tau}$ if and only if it is a weak equivalence in $\operatorname{SPr}\left(\mathrm{Aff}_{\mathscr{V}}\right)$. The functor $\mathbb{R}$ Spec above induces a full embedding

$$
\mathbb{R} \text { Spec: } \operatorname{Ho}\left(\operatorname{Aff}_{\mathscr{V}}\right) \longrightarrow \operatorname{St}(\mathscr{V}, \tau)
$$

Let $A$ be a commutative algebra in $\mathscr{V}$. The category $\operatorname{Mod}(A)$ of $A$-modules is a model category with fibrations and weak equivalences defined by the underlying morphisms in $\mathscr{V}$. In an ideal world, there would be a stack of modules, denoted by QCoh, defined by

$$
\underline{\mathrm{QCoh}}(A)=|w \operatorname{Mod}(A)| .
$$

Moreover, given a commutative algebra morphism $A \rightarrow B$, the induced map

$$
\underline{\mathrm{QCoh}}(A)=|w \operatorname{Mod}(A)| \longrightarrow \underline{\mathrm{QCoh}}(B)=|w \operatorname{Mod}(B)|
$$

would be defined by the change of coefficients functor $-\otimes_{A} B: \operatorname{Mod}(A) \rightarrow \operatorname{Mod}(B)$. However, there are some issues.

The homotopical issue is that the change of coefficients functor $-\otimes_{A} B$ does not preserve weak equivalences, so it cannot define a map as (5-1). This can be solved by restricting to full subcategories of cofibrant objects,

$$
\underline{\mathrm{QCoh}}(A)=\left|w \operatorname{Mod}(A)_{c}\right|,
$$

since $-\otimes_{A} B$ is a left Quillen functor, so it preserves weak equivalences between cofibrant objects. This is not harmful since $\left|w \operatorname{Mod}(A)_{c}\right| \simeq|w \operatorname{Mod}(A)|$. 
The categorical issue is that this construction does not define a functor since, given commutative algebra morphisms $A \rightarrow B \rightarrow C$ and an $A$-module $M$, the natural isomorphism $M \otimes_{A} B \otimes_{B} C \cong M \otimes_{A} C$ is not the identity. Nevertheless, these natural isomorphisms satisfy the coherence condition by Anel in [2, Définition I.56], so the nonfunctor $A \mapsto \operatorname{Mod}(A)$ defines a weak presheaf of model categories, which can be strictified before restricting to weak equivalences and taking nerves; see [2, Section I.2.3.1]. The strictification of QCoh is explicitly described in [26, Section 1.3.7] in terms of categories of quasicoherent modules, hence the name QCoh of this simplicial presheaf.

The geometric issue is to check that the simplicial presheaf QCoh is a stack. This holds in any HAG context, essentially by definition; see [26, Theorem 1.3.7.2].

Given an admissible operad $\mathcal{O}$ in $\mathscr{V}$, in this section we aim at constructing a moduli stack of $\mathcal{O}$-algebras $\operatorname{Alg}_{\mathscr{V}}(\mathcal{O})$. We would like that

$$
\underline{\operatorname{Alg}}_{V}(\mathcal{O})(A)=\left|w \operatorname{Alg}_{\operatorname{Mod}(A)}(\mathcal{O})\right|,
$$

and that given a commutative algebra morphism $A \rightarrow B$, the induced map

$$
\operatorname{Alg}_{V}(\mathcal{O})(A)=\left|w \operatorname{Alg}_{\operatorname{Mod}(A)}(\mathcal{O})\right| \longrightarrow \underline{\operatorname{Alg}}(\mathcal{O})(B)=\left|w \operatorname{Alg}_{\operatorname{Mod}(B)}(\mathcal{O})\right|
$$

were defined by the change of coefficients functor $-\otimes_{A} B$. We would also like that the forgetful functors $\operatorname{Alg}_{\operatorname{Mod}(A)}(\mathcal{O}) \rightarrow \operatorname{Mod}(A)$ defined a morphism of stacks

$$
\xi^{\mathcal{O}}: \underline{\operatorname{Alg}_{V}}(\mathcal{O}) \longrightarrow \underline{\text { QCoh}} .
$$

In order to achieve this goal, we will face the same difficulties as for the definition of QCoh and some other specific issues. We will rely on the homotopy theory of operads developed in $[20 ; 21]$.

First of all, in order the model category of $\mathcal{O}$-algebras in $\operatorname{Mod}(A)$ to be defined, we need to endow $\operatorname{Mod}(A)$ with a model $\mathscr{V}$-algebra structure.

Lemma 5.1 Let $A$ be a commutative algebra in $\mathscr{V}$. The functor

$$
z_{A}=-\otimes A: \mathscr{V} \rightarrow \operatorname{Mod}(A)
$$

endows $\mathscr{C}=\operatorname{Mod}(A)$ with the structure of a combinatorial symmetric model $\mathscr{V}$-algebra in the sense of Definition 2.5. In addition, it satisfies the very strong unit axiom in Definition A.1.

Proof By [26, Assumption 1.1.0.2], $\operatorname{Mod}(A)$ is a combinatorial model category and satisfies the pushout product axiom and the unit axiom. The monoid axiom follows 
from [23, Theorem 4.1(2)]. The very strong unit axiom follows from [26, Assumption 1.1.0.3]; see Remark A.4.

The functor $z_{A}$, which is obviously strong symmetric monoidal, is a left Quillen functor since its right adjoint, the forgetful functor $\operatorname{Mod}(A) \rightarrow \mathscr{V}$, preserves fibrations and weak equivalences. Moreover, $z_{A}$ satisfies the $\mathbb{I}-$ cofibrant axiom by Lemma A.6.

The next step is to show how, given a commutative algebra morphism $A \rightarrow B$, the change of coefficients functor $-\otimes_{A} B: \operatorname{Mod}(A) \rightarrow \operatorname{Mod}(B)$ induces a functor $-\otimes_{A}$ $B: \operatorname{Alg}_{\operatorname{Mod}(A)}(\mathcal{O}) \rightarrow \operatorname{Alg}_{\operatorname{Mod}(B)}(\mathcal{O})$.

Definition 5.2 A symmetric $\mathscr{V}$-algebra functor is a lax symmetric monoidal functor between symmetric $\mathscr{V}$-algebras $F: \mathscr{C} \rightarrow \mathscr{D}$ equipped with a monoidal natural isomorphism $F z_{\mathscr{C}}(X) \cong z_{\mathscr{D}}(X)$.

A symmetric $\mathscr{V}$-algebra Quillen adjunction $F: \mathscr{C} \rightleftarrows \mathscr{D}: G$ is a Quillen pair between symmetric model $\mathscr{V}$-algebras which is a lax-lax symmetric monoidal adjunction and such that $F$ is equipped with the structure of a symmetric $\mathscr{V}$-algebra functor. In particular $F$ is strong monoidal. We assume in addition that the $\mathbb{I}$-cofibrant axiom in $[21$, Definition B.6] is satisfied.

Example 5.3 Given a commutative algebra morphism $A \rightarrow B$ in $\mathscr{V}$, the functor $-\otimes_{A} B: \operatorname{Mod}(A) \rightarrow \operatorname{Mod}(B)$ is the left adjoint in a symmetric $\mathscr{V}$-algebra Quillen adjunction. The natural isomorphism $z_{A}(X) \otimes_{A} B=X \otimes A \otimes_{A} B \cong X \otimes B=z_{B}(X)$ is the obvious one. The $\mathbb{I}$-cofibrant axiom follows from Lemmas A.6 and 5.1. If $A \rightarrow B$ is a weak equivalence then this symmetric $\mathscr{V}$-algebra Quillen adjunction is a Quillen equivalence as a consequence of [26, Assumption 1.1.0.3].

Remark 5.4 Given a symmetric $\mathscr{V}$-algebra Quillen adjunction $F$ : $\mathscr{C} \rightleftarrows \mathscr{D}: G$ and an operad $\mathcal{O}$ in $\mathscr{V}$, there is an induced Quillen pair between categories of algebras $F: \operatorname{Alg}_{\mathscr{C}}(\mathcal{O}) \rightleftarrows \operatorname{Alg}_{\mathscr{D}}(\mathcal{O}): G$ which overlies the previous adjunction; see [21, Proposition 7.1] and its proof. The latter is a Quillen equivalence if the former is and $\mathcal{O}$ is admissible [21, Theorem D.11].

Therefore, if $\mathcal{O}$ is an admissible operad, we can define a simplicial presheaf $\operatorname{Alg}_{\mathscr{V}}(\mathcal{O})$ by

$$
\underline{\operatorname{Alg}}_{\mathscr{V}}(\mathcal{O})(A)=\left|w \operatorname{Alg}_{\operatorname{Mod}(A)}(\mathcal{O})_{c}\right|
$$

Honestly speaking, we should go through a categorical strictification process before restricting to weak equivalences between cofibrant objects and taking nerves, as in the 
case of QCoh, but we will keep this technical issue aside so as not to overload the paper.

Now we must show that $\operatorname{Alg}_{\mathscr{V}}(\mathcal{O})$ is a stack. The most complicated property is descent. We will see that descent for operads follows from descent for modules, which is an assumption of HAG contexts. In order to check this technical condition, we need some definitions.

Definition 5.5 Given a cosimplicial commutative algebra $A^{\bullet}$, a cosimplicial $A^{\bullet}$ module $M^{\bullet}=\left(M^{n}, d^{i}, s^{i}\right)$ consists of a $A^{n}$-module $M^{n}$ for each $n \geq 0$ together with coface morphisms of $A^{n-1}$-modules $d^{i}: M^{n-1} \rightarrow M^{n}$ and codegeneracy morphisms of $A^{n+1}$-modules $s^{i}: M^{n+1} \rightarrow M^{n}, 0 \leq i \leq n$, satisfying the usual cosimplicial identities. Here $M^{n}$ is regarded as $A^{n-1}$-module and as an $A^{n+1}$-module via restriction of scalars along the coface $d^{i}: A^{n-1} \rightarrow A^{n}$ and codegeneracy $s^{i}: A^{n+1} \rightarrow A^{n}$ in $A^{\bullet}$, respectively.

We say that a cosimplicial $A^{\bullet}$-module $M^{\bullet}$ is homotopy cartesian if the derived adjoints $M^{n-1} \otimes_{A^{n-1}}^{\mathbb{L}} A^{n} \rightarrow M^{n}$ and $M^{n+1} \otimes_{A^{n+1}}^{\mathbb{L}} A^{n} \rightarrow M^{n}$ of all coface and codegeneracy maps are weak equivalences.

Remark 5.6 The category $\operatorname{Mod}\left(A^{\bullet}\right)$ of cosimplicial $A^{\bullet}$-modules carries (at least) two combinatorial model structures, called projective and injective, with the same weak equivalences; see Barwick [3, Theorems 2.28 and 2.30 and Section 2.22]. A morphism $f^{\bullet}: M^{\bullet} \rightarrow N^{\bullet}$ of cosimplicial $A^{\bullet}$-modules is a weak equivalence if each $f^{n}: M^{n} \rightarrow N^{n}$ is a weak equivalence. Moreover $f^{\bullet}$ is a projective fibration if each $f^{n}$ is a fibration in $\operatorname{Mod}\left(A^{n}\right)$ (or equivalently in $\mathscr{V}$ ), and $f^{\bullet}$ is an injective cofibration if each $f^{n}$ is a cofibration in $\operatorname{Mod}\left(A^{n}\right)$. The injective model structure is technically more convenient, and will be the only one we use. Toën and Vezzosi use the projective model structure in [26], probably because the injective model structure was not available at that time. We can freely change since both model structures have the same weak equivalences.

We must consider $\mathcal{O}$-algebras in $\operatorname{Mod}\left(A^{\bullet}\right)$. For this, we need the following result.

Lemma 5.7 The injective model structure and the tensor product of $A^{n}$-modules, $n \geq 0$, induce a combinatorial symmetric monoidal model structure on $\operatorname{Mod}\left(A^{\bullet}\right)$ satisfying the very strong unit axiom. Moreover, the functor $z_{A^{\bullet}}: \mathscr{V} \rightarrow \operatorname{Mod}\left(A^{\bullet}\right)$, $z_{A^{\bullet}}(X)^{n}=X \otimes A^{n}$, endows $\operatorname{Mod}\left(A^{\bullet}\right)$ with the structure of a symmetric model q -algebra. 
Proof The pushout product axiom, the monoid axiom, and the very strong unit axiom in $\operatorname{Mod}\left(A^{\bullet}\right)$ follow from the corresponding axioms in $\operatorname{Mod}\left(A^{n}\right)$, checked in Lemma 5.1, since we are working with the injective model structure. Hence $\operatorname{Mod}\left(A^{\bullet}\right)$ is a combinatorial symmetric monoidal model category in the sense of Definition 2.1, since it is locally presentable.

The functor $z_{A} \bullet$ is obviously strong symmetric monoidal. Its right adjoint sends a cosimplicial $A^{\bullet}$-module $M^{\bullet}$ to the $\operatorname{limit}_{\lim \in \Delta} M^{n}$ in $\mathscr{V}$. By Lemma 5.1, each $z_{A^{n}}$ preserves (trivial) cofibrations, hence so does $z_{A}$ • for the injective model structure. Finally, the $\mathbb{I}$-cofibrant axiom for $z_{A} \bullet$ is a consequence of the fact that this axiom is satisfied by all $z_{A^{n}}$; see again Lemma 5.1 .

Notice that the functor $z_{A} \bullet$ sends cofibrant objects to homotopy cartesian simplicial $A^{\bullet}$-modules.

Now we are ready to show that $\operatorname{Alg}_{\mathscr{V}}(\mathcal{O})$ is a stack, at least when $\mathcal{O}$ is cofibrant.

Proposition 5.8 Let $\mathcal{O}$ be a cofibrant operad in $\mathscr{V}$. The simplicial presheaf $\underline{\operatorname{Alg}}_{\mathcal{V}}(\mathcal{O})$ is a stack.

In the proof of descent, we use the following technical observation.

Lemma 5.9 Let $F: \mathscr{A} \rightleftarrows \mathscr{B}: G$ be an adjoint pair $F \dashv G$. The functor $F$ is fully faithful if and only if the unit $X \rightarrow G F(X)$ is a natural isomorphism. Moreover, if $F$ is fully faithful then the essential image of $F$ consists of those objects $Y$ in $\mathscr{B}$ such that the counit $F G(Y) \rightarrow Y$ is an isomorphism.

Proof of Proposition 5.8 By Example 5.3 and Remark 5.4, if $A \stackrel{\sim}{\rightarrow} B$ is a weak equivalence in $\operatorname{Comm}(\mathscr{V})$ then $-\otimes_{A} B: \operatorname{Alg}_{\operatorname{Mod}(A)}(\mathcal{O}) \rightarrow \operatorname{Alg}_{\operatorname{Mod}(B)}(\mathcal{O})$ is the left adjoint of a Quillen equivalence, hence it induces a weak equivalence

$$
\left|w \operatorname{Alg}_{\operatorname{Mod}(A)}(\mathcal{O})_{c}\right| \stackrel{\sim}{\longrightarrow}\left|w \operatorname{Alg}_{\operatorname{Mod}(B)}(\mathcal{O})_{c}\right| .
$$

This proves that $\underline{\operatorname{Alg}}_{\mathscr{V}}(\mathcal{O})$ preserves weak equivalences.

Let $A$ and $B$ be fibrant commutative algebras in $\mathscr{V}$. Then the product category $\operatorname{Mod}(A) \times \operatorname{Mod}(B)$ is a symmetric model $\mathscr{V}$-algebra with the obvious product structure. Moreover, the natural projections induce a symmetric model $\mathscr{V}$-algebra Quillen equivalence [26, proof of Lemma 1.3.2.3(1)],

$$
\operatorname{Mod}(A \times B) \stackrel{\left(-\otimes_{A \times B} A,-\otimes_{A \times B} B\right)}{\longleftarrow} \operatorname{Mod}(A) \times \operatorname{Mod}(B) .
$$


Again by Remark 5.4, we obtain an induced Quillen equivalence between categories of $\mathcal{O}$-algebras,

$$
\operatorname{Alg}_{\operatorname{Mod}(A \times B)}(\mathcal{O}) \rightleftarrows \operatorname{Alg}_{\operatorname{Mod}(A) \times \operatorname{Mod}(B)}(\mathcal{O})=\operatorname{Alg}_{\operatorname{Mod}(A)}(\mathcal{O}) \times \operatorname{Alg}_{\operatorname{Mod}(B)}(\mathcal{O})
$$

The left adjoint gives rise to a weak equivalence

$$
\left|\operatorname{Alg}_{\operatorname{Mod}(A \times B)}(\mathcal{O})_{c}\right| \stackrel{\sim}{\longrightarrow}\left|\operatorname{Alg}_{\operatorname{Mod}(A)}(\mathcal{O})_{c}\right| \times\left|\operatorname{Alg}_{\operatorname{Mod}(B)}(\mathcal{O})_{c}\right| .
$$

Therefore $\operatorname{Alg}_{\mathscr{V}}(\mathcal{O})$ preserves finite homotopy products.

Now, let us tackle the descent condition. Let $A$ be a commutative algebra, that we also regard as a constant cosimplicial object, $B^{\bullet}$ a cosimplicial commutative algebra, and $\varphi: A \rightarrow B^{\bullet}$ a map as in [26, Assumption 1.3.2.2(3)]. There is a symmetric $\mathscr{V}$-algebra Quillen adjunction

$$
\operatorname{Mod}(A) \underset{\varphi^{*}}{\stackrel{\varphi_{*}}{\rightleftarrows}} \operatorname{Mod}\left(B^{\bullet}\right)
$$

defined by $\varphi_{*}(M)^{n}=M \otimes_{A} B^{n}$. This is indeed a Quillen pair because we are using the injective model structure on the right. The derived adjoint pair

$$
\operatorname{Ho} \operatorname{Mod}(A) \underset{\mathbb{R} \varphi^{*}}{\stackrel{\mathbb{L} \varphi_{*}}{\rightleftarrows}} \operatorname{Ho} \operatorname{Mod}\left(B^{\bullet}\right)
$$

satisfies the following properties: $\mathbb{L} \varphi_{*}$ is fully faithful and any homotopy cartesian $B^{\bullet}$-module is in the essential image of $\mathbb{L} \varphi_{*}$ [26, Assumption 1.3.2.2(3)]. These two properties can be read as properties of the unit and counit of the derived adjoint pair; see Lemma 5.9.

By Remark 5.4, the previous symmetric $\mathscr{V}$-algebra Quillen adjunction induces a Quillen adjunction

$$
\operatorname{Alg}_{\operatorname{Mod}(A)}(\mathcal{O}) \underset{\varphi^{*}}{\stackrel{\varphi_{*}}{\rightleftarrows}} \operatorname{Alg}_{\operatorname{Mod}\left(B^{\bullet}\right)}(\mathcal{O})
$$

Moreover, the derived adjunction

$$
\operatorname{Ho} \operatorname{Alg}_{\operatorname{Mod}(A)}(\mathcal{O}) \underset{\mathbb{R} \varphi^{*}}{\stackrel{\mathbb{L} \varphi_{*}}{\rightleftarrows}} \operatorname{Ho~} \operatorname{Alg}_{\operatorname{Mod}\left(B^{\bullet}\right)}(\mathcal{O})
$$

overlies the previous derived adjoint pair, since cofibrant $\mathcal{O}$-algebras in $\operatorname{Mod}(A)$ have an underlying cofibrant $A$-module [21, Corollaries C.3 and D.3] and fibrant $\mathcal{O}$-algebras in $\operatorname{Mod}\left(B^{\bullet}\right)$ have an underlying fibrant cosimplicial $B^{\bullet}$-module. Therefore, the unit and counit of the former derived adjunction underlie those of the latter, so they share 
the properties in the statement of Lemma 5.9. This, together with [26, Corollary B.0.8], finishes the proof.

In order to check that $\operatorname{Alg}_{\mathscr{V}}(\mathcal{O})$ is actually a stack for any admissible operad $\mathcal{O}$, we need functoriality in $\mathcal{O}$ for $\overline{\operatorname{Alg}}_{V}(\mathcal{O})$. Continuing with our ideal picture, given a morphism of admissible operads $\overline{f:} \mathcal{O} \rightarrow \mathcal{P}$, we would like to have a morphism of simplicial presheaves

$$
\underline{\operatorname{Alg}}_{V}(f): \underline{\operatorname{Alg}}_{V}(\mathcal{P}) \longrightarrow \underline{\operatorname{Alg}_{V}}(\mathcal{O})
$$

defined by the contravariant change of base operad functor $f^{*}$ in (4-1),

$$
{\operatorname{A\operatorname {lg}_{V}}}_{\mathcal{P}}(\mathcal{P})(A)=\left|w \operatorname{Alg}_{\operatorname{Mod}(A)}(\mathcal{P})\right| \longrightarrow \underline{\operatorname{Alg}}_{V}(\mathcal{O})(A)=\left|w \operatorname{Alg}_{\operatorname{Mod}(A)}(\mathcal{O})\right|
$$

The functor $f^{*}$ does preserve all weak equivalences. The problem here is that, in order to define the simplicial presheaf $\operatorname{Alg}_{\mathcal{V}}(\mathcal{O})$, we have had to restrict to cofibrant $\mathcal{O}$-algebras (5-2), and $f^{*}$ does not preserve cofibrant objects.

In order to solve this problem, we thicken $\operatorname{Alg}_{\mathscr{V}}(\mathcal{O})$, ie we give an alternative but weakly equivalent definition in terms of nerves of categories of weak equivalences between a class of objects bigger than cofibrant ones.

Let $\operatorname{Alg}_{\operatorname{Mod}(A)}^{p c}(\mathcal{O}) \subset \operatorname{Alg}_{\operatorname{Mod}(A)}(\mathcal{O})$ be the full subcategory of $\mathcal{O}$-algebras whose underlying $A$-module is pseudocofibrant in the sense of [21, Definition A.1]. The definition of pseudocofibrant object is recalled in Remark A.2 below. Cofibrant algebras over an admissible operad $\mathcal{O}$ have a pseudocofibrant underlying $A$-module by [21, Corollary D.3], ie $\operatorname{Alg}_{\operatorname{Mod}(A)}(\mathcal{O})_{c} \subset \operatorname{Alg}_{\operatorname{Mod}(A)}^{p c}(\mathcal{O})$. Moreover, this inclusion induces a weak equivalence on nerves $\left|w \operatorname{Alg}_{\operatorname{Mod}(A)}(\mathcal{O})_{c}\right| \simeq\left|w \operatorname{Alg}_{\operatorname{Mod}(A)}^{p c}(\mathcal{O})\right|$, since functorial cofibrant replacements define a homotopy inverse.

We can equivalently define $\operatorname{Alg}_{\mathscr{V}}(\mathcal{O})$ by

$$
\underline{\operatorname{Alg}}(\mathcal{O})(A)=\left|w \operatorname{Alg}_{\operatorname{Mod}(A)}^{p c}(\mathcal{O})\right|
$$

since, given a commutative algebra morphism $A \rightarrow B$, the change of coefficients functor $-\otimes_{A} B$ preserves pseudocofibrant modules (see [26, Assumption 1.1.0.2] and [21, Remark B.9]), and weak equivalences between them (see Lemma A.6).

The functor $f^{*}$ in (4-1) is the identity on underlying objects, hence it yields a morphism of simplicial presheaves $\underline{\operatorname{Alg}}_{V}(f): \underline{\operatorname{Alg}}_{V}(\mathcal{P}) \rightarrow \underline{\operatorname{Alg}} \mathscr{V}(\mathcal{O})$ given by

$$
\underline{\operatorname{Alg}_{\mathcal{V}}}(\mathcal{P})(A)=\left|w \operatorname{Alg}_{\operatorname{Mod}(A)}^{p c}(\mathcal{P})\right| \longrightarrow \underline{\operatorname{Alg} \mathscr{V}}(\mathcal{O})(A)=\left|w \operatorname{Alg}_{\operatorname{Mod}(A)}^{p c}(\mathcal{O})\right| .
$$

Moreover, this morphism is a weak equivalence if $f$ is a weak equivalence. 
Corollary 5.10 Let $\mathcal{O}$ be an admissible operad in $\mathscr{V}$. The simplicial presheaf $\operatorname{Alg}_{\mathcal{V}}(\mathcal{O})$ is a stack.

Proof Let $f: \widetilde{\mathcal{O}} \stackrel{\sim}{\rightarrow} \mathcal{O}$ be a cofibrant replacement. The morphism of simplicial presheaves $\underline{\operatorname{Alg}}_{V}(f): \operatorname{Alg}_{V}(\mathcal{O}) \rightarrow \underline{\operatorname{Alg}} \sqrt{\mathcal{O}}(\widetilde{\mathcal{O}})$ is a weak equivalence, $\operatorname{and} \underline{\operatorname{Alg}}_{V}(\widetilde{\mathcal{O}})$ is a

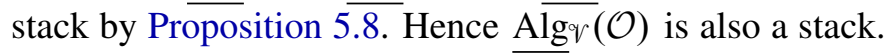

Remark 5.11 By Corollary 4.7, $\operatorname{Alg}_{\mathscr{V}}(\mathcal{O})$, regarded as a contravariant functor in $\mathcal{O}$, takes homotopy colimits of admissible operads to homotopy limits of stacks.

For $\mathcal{O}$ an admissible operad, the forgetful functors $\operatorname{Alg}_{\operatorname{Mod}(A)}(\mathcal{O}) \rightarrow \operatorname{Mod}(A)$ induce a morphism of stacks

$$
\xi^{\mathcal{O}}: \underline{\operatorname{Alg}_{V}}(\mathcal{O}) \longrightarrow \underline{\text { QCoh}} .
$$

Indeed, if $\mathcal{O}(0)$ is cofibrant in $\mathscr{V}$, eg if $\mathcal{O}$ is a cofibrant operad [21, Corollary C.3], we can use the unthickened definition of $\operatorname{A\operatorname {lg}} \mathcal{V}(\mathcal{O})$ to define this morphism by the maps

$$
\underline{\operatorname{Alg}} \underset{V}{(O)}(A)=\left|w \operatorname{Alg}_{\operatorname{Mod}(A)}(\mathcal{O})_{c}\right| \longrightarrow \underline{\mathrm{QCoh}}(A)=\left|w \operatorname{Mod}(A)_{c}\right|,
$$

since the underlying object of a cofibrant $\mathcal{O}$-algebra is cofibrant [21, Corollary D.3]. For a general $\mathcal{O}$, we thicken $\mathrm{QCoh}$ without changing its homotopy type by using the subcategories $\operatorname{Mod}^{p c}(A) \subset \overline{\operatorname{Mod}(A)}$ of pseudocofibrant $A$-modules,

$$
\underline{\mathrm{QCoh}}(A)=\left|w \operatorname{Mod}^{p c}(A)\right| .
$$

Then we define $\xi^{\mathcal{O}}$ by using the thickened versions of the source and the target stacks and the maps

$$
\underline{\operatorname{Alg}_{V}}(\mathcal{O})(A)=\left|w \operatorname{Alg}_{\operatorname{Mod}(A)}^{p c}(\mathcal{O})\right| \longrightarrow \underline{\mathrm{QCoh}}(A)=\left|w \operatorname{Mod}^{p c}(A)_{c}\right|
$$

The morphism $\xi^{\mathcal{O}}$ is natural in the admissible operad $\mathcal{O}$. Our next aim is to study properties of this morphism.

Definition 5.12 Let $\mathcal{O}$ be an admissible operad in $\mathscr{V}, A$ a commutative algebra in $\mathscr{V}$, and $M$ an $A$-module. The stack of $\mathcal{O}$-algebra structures on $M$ is the stack over $\mathbb{R} \operatorname{Spec}(A)$,

$$
\underline{\operatorname{Map}}_{O(\mathscr{V})}\left(\mathcal{O}, \operatorname{End}_{\operatorname{Mod}(A)}(M)\right),
$$

defined as the homotopy pullback of $\xi^{\mathcal{O}}$ along the morphism $g: \mathbb{R} \operatorname{Spec}(A) \rightarrow \underline{\mathrm{QCoh}}$ represented by $M$.

Honestly speaking, we should take a fibrant replacement of QCoh in order the map $g$ to be defined in the category of stacks, not just in the homotopy category. However, we will allow us this kind of language. 
Remark 5.13 For any cofibrant commutative algebra $A$ in $\mathscr{V}, \operatorname{Mod}(A)$ is the base combinatorial symmetric monoidal model category of a new HAG context and $\operatorname{Aff}_{\operatorname{Mod}(A)}^{\sim \tau}$ is Quillen equivalent to the comma model category $\operatorname{Aff}_{\mathscr{V}}^{\sim}, \tau \mid \mathbb{R} \operatorname{Spec}(A)$; see [26, Proposition 1.3.2.10] and its preceding paragraph. Hence, we can identify stacks over $\mathbb{R} \operatorname{Spec}(A)$ with stacks in this new HAG context.

Remark 5.14 By Theorem 4.6, for any $A$-algebra $B$,

$$
\underline{\operatorname{Map}}_{\mathrm{Op}(\mathscr{V})}\left(\mathcal{O}, \operatorname{End}_{\operatorname{Mod}(A)}(M)\right)(B) \simeq \operatorname{Map}_{\mathrm{Op}(\mathscr{V})}\left(\mathcal{O}, \operatorname{End}_{\operatorname{Mod}(B)}\left(\widetilde{M \otimes_{A}^{\mathbb{L} B}}\right)\right),
$$

where $\tilde{N}$ denotes a fibrant-cofibrant replacement of a $B$-module $N$. This justifies the name of this stack.

Remark 5.15 The stack $\operatorname{Map}_{\operatorname{Op}(\mathscr{V})}\left(\mathcal{O}, \operatorname{End}_{\operatorname{Mod}(A)}(M)\right)$ is a contravariant functor in the admissible operad $\mathcal{O}$ since $\operatorname{Alg}_{\mathscr{V}}(\mathcal{O})$ is a contravariant functor in $\mathcal{O}$ and $\xi^{\mathcal{O}}$ is natural. Moreover, it takes homotopy colimits of admissible operads to homotopy limits of stacks, since mapping spaces take homotopy colimits in the first variable to homotopy limits of spaces.

We will show that the stack of $\mathcal{O}$-algebra structures on a module $M$ is representable when $M$ is perfect in the sense of the following definition.

Definition 5.16 [26, Definition 1.2.3.6] Let $Y$ be an object in $\mathscr{V}$. For the sake of simplicity, let us assume that $Y$ is fibrant and cofibrant. Let

$$
q_{X}: Q X \stackrel{\sim}{\rightarrow} X, \quad r_{X}: X \stackrel{\sim}{\rightarrow} R X,
$$

be functorial cofibrant and fibrant replacements in $\mathscr{V}$, respectively. Moreover, denote internal morphism objects in $\mathscr{V}$ by Hom $\mathscr{V}$.

The dual of $Y$ is $Y^{\vee}=\operatorname{Hom}_{V}(Y, R \mathbb{I})$. The object $Y$ is perfect if the composition of the two vertical morphisms in the following diagram is a weak equivalence in $\mathscr{V}$ :

$$
\begin{aligned}
& Y \otimes Y^{\vee}=\operatorname{Hom}_{\mathscr{V}}(\mathbb{I}, Y) \otimes \operatorname{Hom}_{\mathcal{V}}(Y, R \mathbb{I}) \underset{q_{\mathbb{I}}^{*} \otimes \mathrm{id}^{\prime}}{\sim} \operatorname{Hom}_{\mathcal{V}}(Q \mathbb{I}, Y) \otimes \operatorname{Hom}_{\mathcal{V}}(Y, R \mathbb{I}) \\
& \downarrow \otimes \\
& \operatorname{Hom}_{\mathscr{V}}(Q \mathbb{I} \otimes Y, Y \otimes R \mathbb{I}) \\
& \downarrow\left(r_{Y \otimes R \mathbb{I}}\right)_{*} \\
& \operatorname{Hom}_{\mathcal{V}}(Y, Y)=\operatorname{Hom}_{\mathcal{V}}(\mathbb{I} \otimes Y, Y \otimes \mathbb{I}) \stackrel{\sim}{x} \operatorname{Hom}_{V}(Q \mathbb{I} \otimes Y, R(Y \otimes R \mathbb{I})),
\end{aligned}
$$


where $x=\operatorname{Hom}_{V}\left(q_{\mathbb{I}} \otimes \operatorname{id}_{Y}, r_{Y \otimes R \mathbb{I}}\left(\operatorname{id}_{Y} \otimes r_{Y}\right)\right)$. In this case, this diagram yields an isomorphism $Y \otimes Y^{\vee} \cong \operatorname{Hom}_{V}(Y, Y)$ in $\operatorname{Ho}^{\mathscr{V}}$. An arbitrary $Y$ is perfect if a fibrant-cofibrant replacement is perfect.

If $A$ is a commutative algebra, we can replace $\mathscr{V}$ with $\operatorname{Mod}(A)$ and $\mathbb{I}$ with $A$ in the previous paragraphs. This yields the notions of dual and perfect $A$-module.

Theorem 5.17 Let $\mathcal{O}$ be an admissible operad in $\mathscr{V}, A$ a commutative algebra in $\mathscr{V}$ and $M$ a perfect $A$-module. The stack $\operatorname{Map}_{\mathrm{Op}(\mathcal{V})}\left(\mathcal{O}, \operatorname{End}_{\operatorname{Mod}(A)}(M)\right)$ is affine.

We need a technical result in order to prove this theorem. Recall that a sequence is an object in the product model category $\mathscr{V}^{\mathbb{N}}=\prod_{n \geq 0} \mathscr{V}$. There is a Quillen pair

$$
\mathcal{V} \underset{\text { forget }}{\stackrel{\mathcal{F}}{\rightleftarrows}} \mathrm{Op}(\mathscr{V})
$$

where the right adjoint is the forgetful functor $\mathcal{O} \mapsto\{\mathcal{O}(n)\}_{n \geq 0}$ and the left adjoint $\mathcal{F}$ is the free operad functor [20, Section 5].

Lemma 5.18 Any operad in $\mathscr{V}$ is weakly equivalent to the target of a relative cell complex from a free operad on a cofibrant sequence with respect to a set of cofibrations between free operads on cofibrant sequences.

Proof Any operad is weakly equivalent to a cell complex with respect to the set of generating cofibrations in $\mathrm{Op}(\mathscr{V})$. The initial operad is free on a cofibrant sequence: the initial sequence. Generating cofibrations are maps between free operads, actually they are free maps; see the proof of [20, Theorem 1.1]. The underlying sequences of these free operads need not be cofibrant. However, if the tensor unit $\mathbb{I}$ is cofibrant, we can apply the trick at the beginning of the proof of [21, Proposition 4.2] to use cofibrant sequences instead.

If the tensor unit is not cofibrant, the previous trick only allows us to use sequences $U=$ $\{U(n)\}_{n \geq 0}$ with $U(n)$ cofibrant for $n \neq 1$ and $\mathbb{I}$-cofibrant for $n=1$; see [20, Corollary C.3]. Such sequences can be replaced with cofibrant sequences as follows. Let $\tilde{U} \stackrel{\sim}{\rightarrow} U$ be a cofibrant resolution in $\mathscr{V}^{\mathbb{N}}$. The induced morphism $\mathcal{F}(\tilde{U}) \rightarrow \mathcal{F}(U)$ is a weak equivalence by [21, Corollary A.14 and Lemma A.15], since a free operad is built from coproducts of tensor products of the objects in the underlying sequence; see [20, Section 5]. Finally, we can apply the gluing lemma in the cofibration category of operads with underlying pseudocofibrant sequence to replace $\mathcal{F}(U)$ with $\mathcal{F}(\widetilde{U})$; see [21, Proposition C.8 and Corollary 5.2]. 
Proof of Theorem 5.17 By Remark 5.15 and Lemma 5.18, it is enough to prove the theorem for $\mathcal{O}=\mathcal{F}(U)$ a free operad on a cofibrant sequence $U=\left\{U_{n}\right\}_{n \geq 0}$.

We can suppose without loss of generality that $A$ is a cofibrant commutative algebra and $M$ a fibrant-cofibrant $A$-module. We define a stack $F$ over $\mathbb{R} \operatorname{Spec}(A)$ in the sense of Remark 5.13 as follows. Given a commutative $A$-algebra $B \overline{\text {, ie a }}$ morphism of commutative algebras $A \rightarrow B$,

$$
F(B)=\prod_{n \geq 0} \operatorname{Map}_{\operatorname{Mod}(A)}\left(U_{n} \otimes M^{\otimes_{A} n} \otimes_{A} M^{\vee}, B\right) .
$$

This stack is clearly represented by the free commutative $A$-algebra $C$ generated by

$$
\coprod_{n \geq 0} U_{n} \otimes M^{\otimes_{A} n} \otimes_{A} M^{\vee}
$$

Notice that, by adjunction

$$
F(B) \simeq \operatorname{Map}_{\mathrm{Op}(\mathscr{V})}\left(\mathcal{F}(U), \operatorname{End}_{\operatorname{Mod}(B)}\left(\widetilde{M \otimes_{A} B}\right)\right),
$$

where $\overparen{M \otimes_{A} B}$ denotes a fibrant replacement of the cofibrant $B$-module $M \otimes_{A} B$; see [26, Proposition 1.2.3.7] and its preceding paragraphs. However, we cannot define $F$ by the right hand side of the previous equation since it is not functorial in $B$.

Theorem 4.6 shows that, pointwise, $\mathbb{R} \operatorname{Spec}(C) \simeq F$ is the homotopy pullback of $\xi^{\mathcal{F}(U)}$ along $g$. It is only left to define a map of $\operatorname{stacks} \mathbb{R} \operatorname{Spec}(C) \rightarrow \operatorname{Alg} \mathcal{V}(\mathcal{O})$ realizing the pointwise inclusion of the homotopy fiber. One can check that it is enough to take a map determined by an $\mathcal{O}$-algebra structure on the $C$-module $\overline{M \otimes_{A} C}$ corresponding to the connected component of the identity in

$$
\begin{aligned}
\operatorname{Map}_{\mathrm{Op}(\mathscr{V})}\left(\mathcal{F}(U), \operatorname{End}_{\operatorname{Mod}(C)}\left(\widehat{M \otimes_{A} C}\right)\right) & \\
& \simeq F(C) \simeq \mathbb{R} \underline{\operatorname{Spec}}(C)(C)=\operatorname{Map}_{\operatorname{Comm}(\mathscr{V})}(C, C) .
\end{aligned}
$$

One application of Theorem 5.17 is to obtain geometric substacks of $\operatorname{Alg} \mathscr{V}(\mathcal{O})$. The stack QCoh is too big to have nice geometric properties. To this end, it is necessary to restrict to smaller substacks.

Definition 5.19 Given a stack $G$, a substack of $G$ is a stack $F$ such that, for any $X$ in Aff $\mathscr{v}, F(X) \subset G(X)$ is a simplicial subset formed by certain connected components of $G(X)$ and the inclusions define a morphism $F \rightarrow G$.

Example 5.20 Below we give examples of 1-geometric substacks $F \subset \underline{\text { QCoh in }}$ different HAG contexts. In each case, we give a name for $F$ and specify the property that an $A$-module must satisfy so the corresponding component of $\underline{\mathrm{QCoh}}(A) \simeq|w \operatorname{Mod}(A)|$ lies in $F(A)$. 
(1) The substack Vect ${ }_{n}$ of rank $n$ vector bundles, $n \geq 0$, for any HAG context where the tensor unit $\mathbb{I}$ is finitely presented in $\mathscr{V}$ in the sense of [26, Definition 1.2.3.1], and such that all smooth morphisms of commutative algebras belong to $\boldsymbol{P}$; see [26, Corollary 1.3.7.12]. An $A$-module $M$ is a rank $n$ vector bundle if there is a covering $\left\{A \rightarrow A_{i}\right\}_{i \in I}$ in $\tau$ such that each $M \otimes_{A}^{\mathbb{L}} A_{i}$ is weakly equivalent

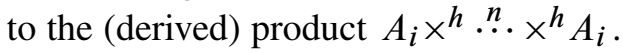

(2) The substack Perf of perfect modules in the sense of Definition 5.16, in the weak HAG context for complicial algebraic geometry [26, 2.3.2]; see [26, Proposition 2.3.3.1].

(3) The substack Perf $[a, b]$ of locally cellular modules of amplitude contained in a finite interval $[a, b]$ in the sense of [26, Definition 2.3.5.2], in the stronger HAG context for complicial algebraic geometry [26, 2.3.4]; see [26, Proposition 2.3.5.4].

Example (1) still holds if $\mathbb{I}$ is not finitely presented but $\boldsymbol{P}$ contains all formally smooth morphisms. Examples (2) and (3) have versions in brave new algebraic geometry; see $[26,2.4 .1]$.

Definition 5.21 Let $\mathcal{O}$ be an admissible operad in $\mathscr{V}$ and $F$ a substack of QCoh. We define the restricted stack of $\mathcal{O}$-algebras $\operatorname{Alg}_{F}(\mathcal{O})$ as the following pullback:

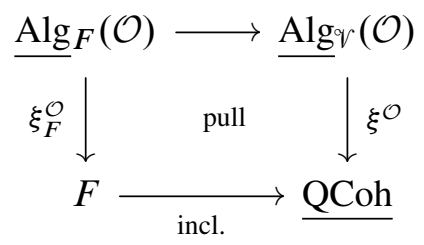

This diagram includes the definition of the map $\xi_{F}^{\mathcal{O}}: \underline{\operatorname{Alg}_{F}}(\mathcal{O}) \rightarrow F$.

Remark 5.22 The pullback square in Definition 5.21 is a homotopy pullback since the bottom horizontal arrow is a fibration of simplicial presheaves for obvious reasons. Therefore $\underline{\operatorname{Alg}_{F}}(\mathcal{O})$ is indeed a stack.

Proposition 5.23 In the conditions of Definition 5.21, if $F$ is $n$-geometric and for any commutative algebra $A$ in $\mathscr{V}$, the connected components of $F(A)$ are represented by perfect $A$-modules, then $\underline{\operatorname{Alg}}_{F}(\mathcal{O})$ is $n$-geometric.

Proof Theorem 5.17 shows that $\xi_{F}^{\mathcal{O}}: \operatorname{Alg}_{F}(\mathcal{O}) \rightarrow F$ is an affine morphism. Hence $\operatorname{Alg}_{F}(\mathcal{O})$ is $n$-geometric by [26, Proposition 1.3.3.4]. 


\section{Spaces and stacks of (unital) $A$-infinity algebras}

Let $\mathscr{V}$ be a symmetric monoidal model category. Assume further that $\mathscr{V}$ is simplicial or complicial, ie a symmetric model $\mathrm{Set}^{\Delta^{\mathrm{op}}}$-algebra or $\mathrm{Ch}(\mathbb{k})$-algebra. Recall from Example 2.3 the canonical map $\phi:$ Ass $\rightarrow$ uAss from the associative operad to the unital associative operad which models the forgetful functor from unital associative algebras to associative algebras. We proved in [19] that $\phi$ is a homotopy epimorphism in $\operatorname{Op}(\mathscr{V})$ in the sense of the following definition; compare [26, Remark 1.2.6.2] and $[19$, Section 2].

Definition 6.1 A map of simplicial sets $g: K \rightarrow L$ is a homotopy monomorphism if it corestricts to a weak equivalence between $K$ and a subset of connected components of $L$. This is equivalent to say that the homotopy fibers of $g$ are empty or weakly contractible.

A morphism $f: X \rightarrow Y$ in a model category $\mathcal{M}$ is a homotopy epimorphism if for any object $Z$ in $\mathcal{M}$ the induced map $f^{*}: \operatorname{Map}_{\mathcal{M}}(Y, Z) \rightarrow \operatorname{Map}_{\mathcal{M}}(X, Z)$ is a homotopy monomorphism.

A map $f: X \rightarrow Y$ is a homotopy monomorphism in $\mathcal{M}$ if it is a homotopy epimorphism in $M^{\mathrm{op}}$. This is the same as saying that the derived codiagonal, which is the map $\Delta=\left(\begin{array}{l}1_{X} \\ 1_{X}\end{array}\right): X \rightarrow X \times \times_{Y}^{h} X$ to the homotopy product of $X$ with itself over $Y$, is a weak equivalence.

The two notions of homotopy monomorphism of simplicial sets coincide.

In particular, if $\mathscr{C}$ is a model $\mathscr{V}$-algebra and $Y$ is a fibrant-cofibrant object in $\mathscr{b}$, the map

$$
\phi^{*}: \operatorname{Map}_{\mathrm{Op}(\mathscr{V})}\left(\mathrm{uAss}_{\mathrm{And}} \operatorname{End}_{\mathscr{C}}(Y)\right) \longrightarrow \operatorname{Map}_{\mathrm{Op}(\mathscr{V})}\left(\operatorname{Ass}, \operatorname{End}_{\mathscr{C}}(Y)\right)
$$

is a homotopy monomorphism.

Proposition 6.2 The map $\mid w \operatorname{Alg}_{\mathscr{C}}$ (uAss) $|\rightarrow| w \operatorname{Alg}_{\mathscr{C}}$ (Ass) $\mid$ induced by the forgetful functor is a homotopy monomorphism.

This result is a consequence of Theorem 4.6 and Lemma 6.3 below. It also follows from [16, Theorem 5.2.3.5], which actually goes further, characterizing the essential image; see Proposition 6.6 below. 


\section{Lemma 6.3 Let}

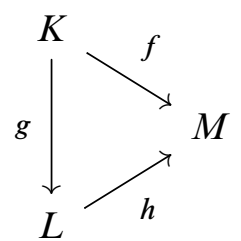

be a commutative triangle of simplicial sets. Denote by $F_{f, x}$ and $F_{h, x}$ the homotopy fibers of $f$ and $h$ at $x \in M_{0}$, respectively. The following statements are equivalent.

(1) The map $g$ is a homotopy monomorphism.

(2) For any $x \in M_{0}$ the map $g_{x}: F_{f, x} \rightarrow F_{h, x}$ induced by $g$ is a homotopy monomorphism.

Proof The square

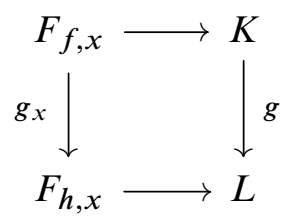

is a homotopy pullback. This lemma follows from the fact that parallel arrows in a homotopy pullback have essentially the same homotopy fibers. Let us spell out what this means in this case. We are interested in the vertical arrows. The homotopy fiber of $g_{x}$ at a vertex $y$ of $F_{h, x}$ coincides with the homotopy fiber of $g$ at the image of $y$ along $F_{h, x} \rightarrow L$. Moreover, the homotopy fiber of $g$ at a vertex $z$ of $L$ coincides with the homotopy fiber of $g_{h(z)}$ at any vertex $y$ of $F_{h, h(z)}$ which maps to the same component as $z$ in $L$.

The image of the injective map $\pi_{0} \mid w \operatorname{Alg}_{\mathscr{C}}$ (uAss) $\left|\hookrightarrow \pi_{0}\right| w \operatorname{Alg}_{\mathscr{C}}$ (Ass) $\mid$ has a friendly characterization.

Definition 6.4 An associative algebra $X$ in $\mathscr{C}$ with underlying fibrant-cofibrant object is quasiunital if there exists a map $u: \widetilde{\mathbb{I}}_{\mathscr{C}} \rightarrow X$, where $q_{\mathbb{I}_{\mathscr{C}}}: \widetilde{\mathbb{I}}_{\mathscr{C}} \stackrel{\sim}{\rightarrow} \mathbb{I}_{\mathscr{C}}$ is a cofibrant replacement of the tensor unit in $\mathscr{b}$ that we do not require to be a fibration, such that the maps

$$
\begin{aligned}
& \tilde{\mathbb{I}}_{\mathscr{C}} \otimes X \stackrel{u \otimes X}{\longrightarrow} X \otimes X \stackrel{\text { mult. }}{\longrightarrow} X, \\
& X \otimes \tilde{\mathbb{I}}_{\mathscr{C}} \stackrel{X \otimes u}{\longrightarrow} X \otimes X \stackrel{\text { mult. }}{\longrightarrow} X,
\end{aligned}
$$

are homotopic to $\tilde{\mathbb{I}}_{\mathscr{C}} \otimes X \stackrel{q_{\mathbb{I}_{\mathscr{C}}} \otimes X}{\sim} \mathbb{I}_{\mathscr{C}} \otimes X \cong X$ and $X \otimes \tilde{\mathbb{I}}_{\mathscr{C}} \stackrel{X \otimes q_{\mathbb{I}_{\mathscr{C}}}}{\longrightarrow} X \otimes \mathbb{I}_{\mathscr{C}} \cong X$, respectively. 
If the associative algebra $X$ does not have an underlying fibrant-cofibrant object, we say that $X$ is quasiunital if a (and hence any) fibrant-cofibrant replacement of $X$ in $\operatorname{Alg}_{\mathscr{C}}$ (Ass) is quasiunital. Such a fibrant-cofibrant replacement has an underlying fibrant-cofibrant object in $\mathscr{C}$ by [21, Corollary D.3].

Remark 6.5 An associative algebra in $\mathscr{C}$ with underlying fibrant-cofibrant object satisfies the condition of being quasiunital with respect to some cofibrant replacement $q_{\mathbb{I}}$ of the tensor unit in $\mathscr{b}$ if and only if it satisfies that condition with respect to any such cofibrant replacement.

Proposition 6.6 The image of $\pi_{0} \mid w \operatorname{Alg}_{\mathscr{C}}$ (uAss) $\left|\hookrightarrow \pi_{0}\right| w \operatorname{Alg}_{\mathscr{C}}$ (Ass) $\mid$ consists of the connected components of quasiunital associative algebras.

This proposition follows from [16, Theorem 5.2.3.5], which is a deep and complicated result. We have not found any elementary proof for this proposition. There is an elementary proof in case $\mathscr{V}=\mathscr{C}=\mathrm{Ch}(\mathbb{k})$ is the category of chain complexes over a field $\mathbb{k}$ and $z$ is the identity functor. This proof uses the existence of minimal models for $A$-infinity algebras, compare [19, Remark 6.8]. The result for $\mathbb{k}$ an arbitrary commutative ring, first proved by Lyubashenko in [17], is already very complicated.

We obtain as a corollary a similar characterization of the image of $\pi_{0}(6-1)$. Recall that vertices in $\operatorname{Map}_{\mathrm{Op}(\mathscr{V})}\left(\mathrm{Ass}_{\mathrm{s}} \operatorname{End}_{\mathscr{C}}(Y)\right)$ do not correspond to associative algebra structures on $Y$, but to $A$-infinity algebra structures, ie algebra structures over a cofibrant resolution $\mathrm{A}_{\infty} \stackrel{\sim}{\rightarrow}$ Ass in $\mathrm{Op}(\mathscr{V})$ of the associative operad Ass.

Corollary 6.7 Let $Y$ be a fibrant-cofibrant object in $\mathscr{C}$. The image of the injective map $\pi_{0} \operatorname{Map}_{\mathrm{Op}(\mathscr{V})}\left(\mathrm{uAss}_{\mathrm{A}} \operatorname{End}_{\mathscr{C}}(Y)\right) \hookrightarrow \pi_{0} \operatorname{Map}_{\mathrm{Op}(\mathscr{V})}\left(\operatorname{Ass}_{\mathrm{s}}, \operatorname{End}_{\mathscr{C}}(Y)\right)$ consists of the connected components of $A$-infinity algebra structures on $Y$ which are weakly equivalent in $\operatorname{Alg}_{\mathscr{b}}\left(\mathrm{A}_{\infty}\right)$ to a quasiunital associative algebra.

This result follows from Proposition 6.6, Theorem 4.6 and an elementary computation with the low-dimensional part of the long homotopy exact sequence of a homotopy fibration.

Remark 6.8 Since we can strictify algebras over cofibrant resolutions of admissible operads by using the Quillen equivalence (4-1), Corollary 6.7 gives a positive answer to the question raised in [19, Remark 7.5].

Quasiunital associative algebras can be characterized in terms of operads. 
Definition 6.9 Given an object $U$ in $\mathscr{V}$ and an integer $m \geq 0$, denote $U[m]$ the sequence of objects in $\mathscr{V}$ whose $m^{\text {th }}$ term is $U$ and whose other terms are the initial object $\varnothing$. This construction defines a functor $\mathscr{V} \rightarrow \mathscr{V}^{\mathbb{N}}$, left adjoint to $\{V(n)\}_{n \geq 0} \mapsto V(m)$.

Let $q_{\mathbb{I}_{V}}: \widetilde{\mathbb{I}}_{\mathscr{V}} \stackrel{\sim}{\rightarrow} \mathbb{I}_{\mathscr{V}}$ be a cofibrant replacement of the tensor unit in $\mathscr{V}$, that we do not require to be a fibration. Choose a cylinder for $\widetilde{\mathbb{I}}_{\mathscr{V}}$, which is a factorization of the folding map

$$
\widetilde{\mathbb{I}}_{\mathscr{V}} \amalg \widetilde{\mathbb{I}}_{\mathscr{V}} \stackrel{i}{\rightarrow} C \stackrel{p}{\underset{\sim}{\longrightarrow}} \widetilde{\mathbb{I}}_{\mathscr{V}} .
$$

The operad Ass ${ }^{q u}$ is defined as the following pushout in $\mathrm{Op}(\mathscr{V})$ :

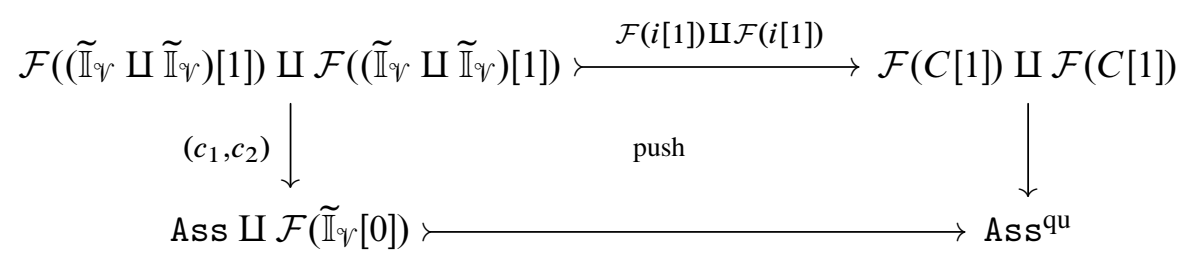

The morphism $c_{1}$ is defined by a map $\left(c_{11}, c_{12}\right): \widetilde{\mathbb{I}}_{V} \amalg \tilde{\mathbb{I}}_{\mathscr{V}} \rightarrow\left(\operatorname{Ass} \amalg \mathcal{F}\left(\tilde{\mathbb{I}}_{\mathscr{V}}[0]\right)\right)(1)$. The map $c_{11}$ is the composite

$$
\tilde{\mathbb{I}}_{\mathscr{V}} \stackrel{q_{\mathbb{I}_{\mathscr{V}}}}{\longrightarrow} \mathbb{I}_{\mathscr{V}}=\operatorname{Ass}(1) \longrightarrow\left(\operatorname{Ass} \amalg \mathcal{F}\left(\tilde{\mathbb{I}}_{\mathscr{V}}[0]\right)\right)(1),
$$

where the second map is given by the inclusion of the first factor of the coproduct. The map $c_{12}$ is the composite:

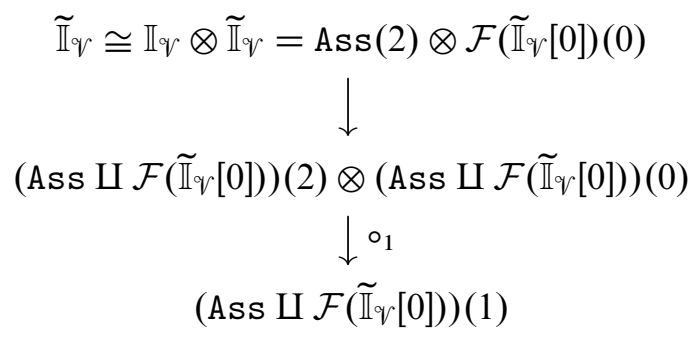

Here the first arrow is given by the tensor product of the inclusions of the factors of the coproduct. Similarly $c_{2}$ is defined by a map $\left(c_{21}, c_{22}\right)$ such that $c_{21}=c_{11}$ and $c_{22}$ is defined like $c_{12}$ but replacing $\circ_{1}$ with $\circ_{2}$.

Remark 6.10 Since $\tilde{\mathbb{I}}_{\mathscr{V}}$ is cofibrant, the inclusion Ass $\succ$ Ass $\amalg \mathcal{F}\left(\tilde{\mathbb{I}}_{\mathscr{V}}[0]\right)$ of the first factor is a cofibration, and so is the composite Ass $\longrightarrow A s s^{q u}$. In particular, Ass ${ }^{q u}$ is admissible by [21, Corollary C.2]. More precisely, $\mathrm{Ass}^{\mathrm{qu}}(0)$ is cofibrant and $\mathrm{Ass}^{\mathrm{qu}}(n)$ is $\mathbb{I}_{\mathscr{V}}$-cofibrant for all $n>0$. 
Remark 6.11 An Ass ${ }^{\text {qu }}$-algebra in $\mathscr{C}$ consists of an associative algebra $X$ in $\mathscr{C}$ together with a map $u: z\left(\tilde{\mathbb{I}}_{\mathscr{V}}\right) \rightarrow X$ and two maps $z(C) \otimes X \rightarrow X$. These maps must satisfy the following two conditions. The restriction of the first $z(C) \otimes X \rightarrow X$ to $z\left(\widetilde{\mathbb{I}}_{\mathscr{V}}\right) \otimes X \amalg z\left(\widetilde{\mathbb{I}}_{\mathscr{V}}\right) \otimes X \cong z\left(\tilde{\mathbb{I}}_{\mathscr{V}} \amalg \tilde{\mathbb{I}}_{\mathscr{V}}\right) \otimes X$ along $z(i) \otimes X$ must coincide with the following pair of maps:

$$
\begin{gathered}
z\left(\tilde{\mathbb{I}}_{\mathscr{V}}\right) \otimes X \stackrel{u \otimes X}{\longrightarrow} X \otimes X \stackrel{\text { mult. }}{\longrightarrow} X \\
z\left(\tilde{\mathbb{I}}_{\mathscr{V}}\right) \otimes X \stackrel{z\left(q_{\mathbb{I}_{\mathscr{V}}}\right) \otimes X}{\sim} z\left(\mathbb{I}_{\mathscr{V}}\right) \otimes X \cong \mathbb{I}_{\mathscr{V}} \otimes X \cong X
\end{gathered}
$$

Moreover, the restriction of the second $z(C) \otimes X \rightarrow X$ must coincide with the following pair of maps:

$$
\begin{gathered}
z\left(\tilde{\mathbb{I}}_{\mathscr{V}}\right) \otimes X \stackrel{\xi}{\cong} X \otimes z\left(\tilde{\mathbb{I}}_{\mathscr{V}}\right) \stackrel{X \otimes u}{\longrightarrow} X \otimes X \stackrel{\text { mult. }}{\longrightarrow} X \\
z\left(\tilde{\mathbb{I}}_{\mathscr{V}}\right) \otimes X \stackrel{\xi}{\cong} X \otimes z\left(\tilde{\mathbb{I}}_{\mathscr{V}}\right) \stackrel{X \otimes z\left(q_{\mathbb{I}_{V}}\right)}{\sim} X \otimes z\left(\mathbb{I}_{\mathscr{V}}\right) \cong X \otimes \mathbb{I}_{\mathscr{V}} \cong X
\end{gathered}
$$

Lemma 6.12 Let $X$ be an associative algebra in $\mathscr{b}$ with underlying fibrant-cofibrant object. Then $X$ is quasiunital if and only if the map Ass $\rightarrow \operatorname{End}_{\mathscr{C}}(X)$ defining the associative algebra structure factors as $\operatorname{Ass}_{\mathrm{s}} \longrightarrow \operatorname{Ass}^{\mathrm{qu}} \rightarrow \operatorname{End}_{\mathscr{C}}(X)$.

Proof Let $q_{\mathbb{I}_{V}}$ be a choice of cofibrant replacement for the tensor unit in $\mathscr{V}$, that we use to define the operad Ass ${ }^{q u}$ in Definition 6.9. According to Remark 6.5, we can take $q_{\mathbb{I}_{\complement}}=z\left(q_{\mathbb{I}_{V}}\right)$ in Definition 6.4.

Suppose $X$ is an Ass ${ }^{q u}$-algebra. Then the underlying associative algebra is quasiunital. Indeed, the map $u$ in Remark 6.11 satisfies the properties in Definition 6.4, since $z(C) \otimes X$ is a cylinder for $z\left(\tilde{\mathbb{I}}_{\mathscr{V}}\right) \otimes X \cong X \otimes z\left(\tilde{\mathbb{I}}_{\mathscr{V}}\right)$ and the maps $z(C) \otimes X \rightarrow X$ in Remark 6.11 are explicit homotopies between the maps that Definition 6.4 demands to be homotopic.

Conversely, suppose that $X$ is a quasiunital associative algebra. Choose a particular $u$ satisfying Definition 6.4. Since $z(C) \otimes X$ is a cylinder for $z\left(\tilde{\mathbb{I}}_{\mathscr{V}}\right) \otimes X \cong X \otimes z\left(\tilde{\mathbb{I}}_{\mathscr{V}}\right)$ we can choose homotopies $z(C) \otimes X \rightarrow X$ between the maps that Definition 6.4 requires to be homotopic. Such homotopies together with $u$ extend the associative algebra structure of $X$ to an Ass ${ }^{\text {qu }}$-algebra structure, according to Remark 6.11.

The universal property of a pushout shows the existence of a unique map

$$
\psi: \mathrm{Ass}^{\mathrm{qu}} \longrightarrow \mathrm{uAss}
$$

such that the composite Ass $\longrightarrow$ Ass ${ }^{q u} \stackrel{\psi}{\rightarrow}$ uAss is $\phi$, defined by

$$
\tilde{\mathbb{I}}_{\mathscr{V}} \stackrel{q_{\mathbb{I}_{V}}}{\sim} \mathbb{I}_{\mathscr{V}}=\mathrm{uAss}(0), \quad C \stackrel{p}{\stackrel{p}{\sim}} \tilde{\mathbb{I}}_{\mathscr{V}} \stackrel{q_{\mathbb{I}_{V}}}{\longrightarrow} \mathbb{I}_{\mathscr{V}}=\operatorname{uAss}(1),
$$


on both copies of $C$.

Proposition 6.13 For any fibrant-cofibrant object $Y$ in $\mathscr{C}$, the map

$$
\psi^{*}: \operatorname{Map}_{\mathrm{Op}(\mathscr{V})}\left(\mathrm{uAss} \operatorname{End}_{\mathscr{C}}(Y)\right) \longrightarrow \operatorname{Map}_{\mathrm{Op}(\mathscr{V})}\left(\operatorname{Ass}^{\mathrm{qu}}, \operatorname{End}_{\mathscr{C}}(Y)\right)
$$

admits a retraction in the homotopy category of simplicial sets.

Proof The composite

$$
\begin{gathered}
\operatorname{Map}_{\mathrm{Op}(\mathscr{V})}\left(\operatorname{uAss}^{*}, \operatorname{End}_{\mathscr{C}}(Y)\right) \\
\operatorname{Map}_{\mathrm{Op}(\mathscr{V})}\left(\operatorname{Ass}^{\mathrm{qu}}, \operatorname{End}_{\mathscr{C}}(Y)\right) \\
\operatorname{Map}_{\mathrm{Op}(\mathscr{V})}\left(\operatorname{Ass}, \operatorname{End}_{\mathscr{C}}(Y)\right)
\end{gathered}
$$

is the homotopy monomorphism in (6-1), which it induces a weak equivalence between the source and a subset of connected components of the target. By Corollary 6.7 and Lemma 6.12, the image of the second map lies on those connected components. Here we use that we can strictify algebras over cofibrant resolutions of admissible operads by using the Quillen equivalence (4-1). Hence we are done.

Now, let us place ourselves in a HAG context, as in the previous section. The notion of substack in Definition 5.19 is too strict, since it is not homotopy invariant. The following lemma shows that homotopy monomorphisms of stacks are homotopy invariant replacements of inclusions of substacks.

Lemma 6.14 Given a morphism of stacks $f: F \rightarrow G$, the following statements are equivalent:

(1) $f(A): F(A) \rightarrow G(A)$ is a homotopy monomorphism of simplicial sets for any commutative algebra $A$ in $\mathscr{V}$.

(2) $f$ is a homotopy monomorphism in $\operatorname{SPr}\left(\operatorname{Aff}_{\mathscr{V}}\right)$.

(3) $f$ is a homotopy monomorphism in $\mathrm{Aff}_{\mathscr{V}}^{\sim, \tau}$.

Proof Homotopy limits in $\operatorname{SPr}\left(\mathrm{Aff}_{\mathscr{V}}\right)$ are computed pointwise, hence (1) $\Leftrightarrow$ (2) follows. The equivalence $(2) \Leftrightarrow(3)$ is a consequence of the fact that the homotopy product $F \times_{G}^{h} F$ in $\operatorname{Aff}_{\mathscr{V}}^{\sim, \tau}$ coincides with the corresponding homotopy product in $\operatorname{SPr}\left(\mathrm{Aff}_{\mathscr{V}}\right)$. 
Recall from the previous section that $\phi$ : Ass $\rightarrow$ uAss induces a morphism of stacks

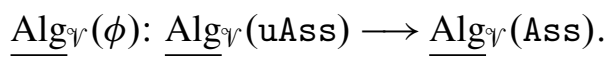

Moreover, if $A$ is a commutative algebra and $M$ is an $A$-module, $\phi$ also induces a morphism of stacks

$$
\phi^{*}: \underline{\operatorname{Map}}_{\mathrm{Op}(\mathscr{V})}\left(\mathrm{uAss}, \operatorname{End}_{\operatorname{Mod}(A)}(M)\right) \longrightarrow \underline{\operatorname{Map}} \underline{\operatorname{Mp}(\mathscr{V})}\left(\operatorname{Ass}, \operatorname{End}_{\operatorname{Mod}(A)}(M)\right) .
$$

Proposition 6.15 The homotopy pullback of the morphism $\operatorname{Alg}_{V}(\phi)$ along any map $g: \mathbb{R S p e c}(A) \rightarrow \operatorname{Alg}_{V}$ (Ass) represented by an associative algebra with underlying perfect $A$-module $\bar{M}$ is an affine stack.

Proof The homotopy pullback of $\xi^{\text {Ass }}: \operatorname{Alg}_{\mathscr{V}}($ Ass $) \rightarrow$ QCoh along the composite $\xi^{\text {Ass }} g$ is the affine stack $\underline{\operatorname{Map}}_{O p(V)}\left(\operatorname{Ass}, \overline{\operatorname{End}}_{\operatorname{Mod}(A)}(M)\right)$. The homotopy pullback

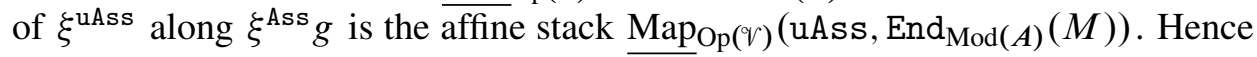

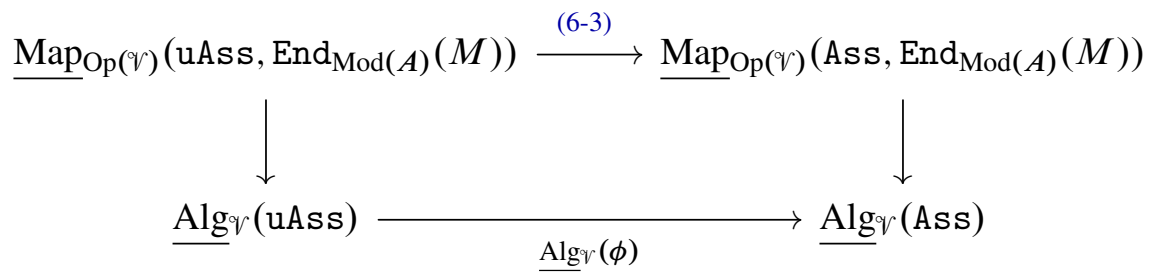

is a homotopy pullback, and the homotopy pullback of $\operatorname{Alg}_{\mathscr{V}}(\phi)$ along $g$ coincides with the homotopy pullback of (6-3) along $\mathbb{R S p e c}(A) \rightarrow \overline{\operatorname{Map}}_{\mathrm{Op}(\mathscr{V})}\left(\operatorname{Ass}, \operatorname{End}_{\operatorname{Mod}(A)}(M)\right)$, the morphism defined by the universal property of a homotopy pullback. Now, the result follows from the fact that a homotopy pullback of affine stack is affine.

Assume from now on that the base category $\mathscr{V}$ of our HAG context is simplicial or complicial.

Theorem 6.16 The morphisms (6-2) and (6-3) are homotopy monomorphisms of stacks.

This result follows from Lemma 6.14, Proposition 6.2, and the fact that (6-1) is a homotopy monomorphism.

Definition 6.17 [26, Definition 1.2.3.1] An object $X$ in a model category $M$ is homotopically finitely presented if the mapping space functor $\operatorname{Map}_{\mu}(X,-)$ preserves filtered homotopy colimits. A morphism $X \rightarrow Y$ in $\mathcal{M}$ is homotopically finitely presented if it is homotopically finitely presented as an object in the comma model category $X \downarrow M$. 
Retracts of homotopically finitely presented objects are also homotopically finitely presented.

Theorem 6.18 Assume that $\mathscr{V}$ is compactly generated in the sense of [26, Definition 1.2.3.4(3)] and that the tensor unit is homotopically finitely presented. Suppose $M$ is a perfect $A$-module. Then the morphism of affine stacks (6-3) is represented by a homotopically finitely presented morphism of commutative $A$-algebras.

Assuming that $\mathscr{V}$ is compactly generated is not a very strong hypothesis. It is satisfied by any model category which is locally finitely presentable (see Adámek and Rosický [1, Definition 1.9]) and finitely generated (see [13, Definition 2.1.17]). All the underlying model categories of the HAG contexts considered in [26] satisfy these properties. Neither is very strong to assume that the tensor unit is homotopically finitely presented. This assumption is also used in [26] to show that the general linear group is a Zariski open affine substack of the affine stack of matrices in a HAG context; see [26, Propositions 1.2.9.4, 1.3.7.10].

In the proof of Theorem 6.18, we use the following proposition.

Proposition 6.19 For any commutative algebra $A$ and any $A$-module $M$, the map

$$
\psi^{*}: \underline{\operatorname{Map}}_{\mathrm{Op}(\mathscr{V})}\left(\mathrm{uAss}, \operatorname{End}_{\operatorname{Mod}(A)}(M)\right) \longrightarrow \underline{\operatorname{Map}} \mathrm{Op(V)}\left(\operatorname{Ass}^{\mathrm{qu}}, \operatorname{End}_{\operatorname{Mod}(A)}(M)\right)
$$

admits a retraction in the homotopy category of stacks.

This result follows in the same way as Proposition 6.13, using Remark 5.14.

Proof of Theorem 6.18 We can suppose without loss of generality that $A$ and $M$ are fibrant and cofibrant. By Definition 6.9, Remark 5.15 and the proof of Theorem 5.17, if $B$ is a fibrant and cofibrant commutative $A$-algebra representing

$$
\underline{\operatorname{Map}}_{\mathrm{Op}(\mathscr{V})}\left(\operatorname{Ass}, \operatorname{End}_{\operatorname{Mod}(A)}(M)\right)
$$

then a commutative $A$-algebra $D$ representing the affine stack

$$
\underline{\operatorname{Map}}_{\mathrm{Op}(\mathscr{V})}\left(\operatorname{Ass}^{\mathrm{qu}}, \operatorname{End}_{\operatorname{Mod}(A)}(M)\right)
$$

can be defined as a certain homotopy pushout

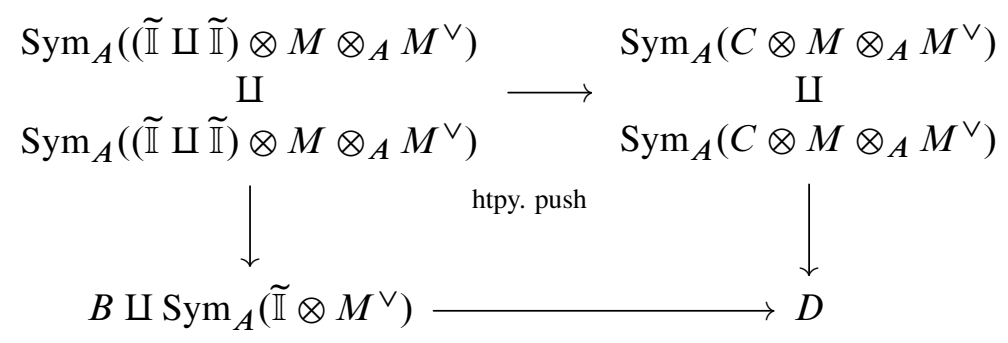


in the category of commutative $A$-algebras. Here $\operatorname{Sym}_{A}$ denotes the free commutative $A$-algebra functor.

Since $\mathscr{V}$ is compactly generated, derived change of coefficient functors along commutative algebra morphisms preserve homotopically finitely presented objects by [26, Proposition 1.2.3.5]. Since $\mathbb{I}$ is homotopically finitely presented in $\mathscr{V}$, then so is $A$ in $\operatorname{Mod}(A)$ and more generally any perfect $A$-module; see [26, Proposition 1.2.3.7]. Therefore all free commutative $A$-algebras in the previous homotopy pushout are homotopically finitely presented. Hence, the map $B \rightarrow D$ is homotopically finitely presented.

By Proposition 6.19, any $B$-algebra representing $\operatorname{Map}_{\mathrm{Op}(\mathscr{V})}\left(\mathrm{uAss}, \operatorname{End}_{\operatorname{Mod}(A)}(M)\right)$ is a homotopy retract of $D$, hence also homotopically finitely presented.

Corollary 6.20 The homotopy pullback of $\operatorname{Alg}_{\mathscr{V}}(\phi)$ along any $g: \mathbb{R} \operatorname{Spec}(A) \rightarrow$ $\mathrm{Alg}_{\mathcal{V}}$ (Ass) represented by an associative algebra with underlying perfect $\overline{A-\text { module } M}$ $\overline{i s}$ an affine stack represented by a homotopically finitely presented $A$-algebra.

Proof In the proof of Proposition 6.15 we showed that the homotopy pullback in the statement can be obtained as a homotopy pullback along (6-3). Hence this result follows from Theorem 6.18 and the fact that the cobase change of a homotopically finitely presented morphism is homotopically finitely presented [26, Proposition 1.2.3.3(3)].

We end this section with an example showing that the affine stack of associative algebra structures $\operatorname{Map}_{\mathrm{Op}(\mathscr{V})}\left(\operatorname{Ass}, \operatorname{End}_{\operatorname{Mod}(A)}(M)\right)$ need not be homotopically finitely presented over $\mathbb{R} \underline{\operatorname{Spec}(\mathbb{I})}$, even for $M$ perfect. This is a big contrast with the classical situation.

Let us place ourselves in any of the two complicial algebraic geometry contexts in [26, Section 2.3]. We will use homological notation for complexes $X_{*}$, so differentials have degree $-1, d: X_{n} \rightarrow X_{n-1}$, ie our complexes are chain complexes, not cochain complexes. We write $|x|=n$ if $x \in X_{n}$.

Consider $\mathbb{k}=\mathbb{Q}, A=\mathbb{Q}$ and $M=\Sigma^{m} \mathbb{Q}$ the $m$-fold suspension of $\mathbb{Q}$. Denote $e$ the degree $m$ generator of $\Sigma^{m} \mathbb{Q}$. In order to compute a commutative differential graded algebra (CDGA) representing the affine stack $\operatorname{Map}_{\mathrm{Op}(\mathrm{Ch}(\mathbb{Q}))}\left(\operatorname{Ass}, \operatorname{End}_{\mathrm{Ch}(\mathbb{Q})}\left(\Sigma^{m} \mathbb{Q}\right)\right)$ we need a cofibrant resolution of Ass in $\operatorname{Op}(\overline{\operatorname{Ch}(\mathbb{Q})})$. The following operad is a well-known cofibrant resolution.

Definition 6.21 The differential graded A-infinity operad $\mathrm{A}_{\infty}$ is defined as follows. The underlying graded operad of $\mathrm{A}_{\infty}$ is freely generated by

$$
\mu_{n} \in \mathrm{A}_{\infty}(n)_{n-2}, \quad n \geq 2,
$$


and the differential is given by

$$
d\left(\mu_{n}\right)=\sum_{\substack{p+q-1=n \\ 1 \leq i \leq p}}(-1)^{q p+(q-1) i} \mu_{p} \circ_{i} \mu_{q} .
$$

The operad $A_{\infty}$ is Stasheff's operad [24]. It is fibrant and cofibrant, and the morphism $\mathrm{A}_{\infty} \rightarrow$ Ass, defined by $\mu_{2} \mapsto 1 \in \mathbb{Q}=\operatorname{Ass}(2)$ and $\mu_{n} \mapsto 0, n>2$, is a trivial fibration.

Let $A$ be any CDGA. An $\mathrm{A}_{\infty}$-algebra structure on the $A$-module $\Sigma^{m} \mathbb{Q} \otimes_{\mathbb{Q}} A \cong \Sigma^{m} A$ is determined by degree $n-2$ morphisms of graded $\mathbb{Q}$-modules,

$$
\mu_{n}: \Sigma^{m} \mathbb{Q} \otimes_{\mathbb{Q}} \stackrel{n}{n} \otimes_{\mathbb{Q}} \Sigma^{m} \mathbb{Q} \longrightarrow \Sigma^{m} \mathbb{Q} \otimes_{\mathbb{Q}} A, \quad n \geq 2,
$$

satisfying certain equations. These morphisms are determined by the structure constants in $A$,

$$
x_{n}, \quad\left|x_{n}\right|=n-2+m n-m, \quad n \geq 2,
$$

such that

$$
\mu_{n}(e, . \stackrel{n}{.}, e)=x_{n} e .
$$

One can straightforwardly check that a choice of structure constants determines an $\mathrm{A}_{\infty}$-algebra structure if and only if the following equations hold in $A$,

$$
d\left(x_{n}\right)=\sum_{\substack{p+q-1=n \\ 1 \leq i \leq p}}(-1)^{(q-1) i+(p+m(i-1))(q+1) m} x_{q} x_{p}, \quad n \geq 2 .
$$

Hence we deduce the following result.

Proposition 6.22 The stack $\underline{\operatorname{Map}}_{\mathrm{Op}(\mathrm{Ch}(\mathbb{Q}))}\left(\mathrm{Ass}, \operatorname{End}_{\mathrm{Ch}(\mathbb{Q})}\left(\Sigma^{m} \mathbb{Q}\right)\right)$ is isomorphic to $\mathbb{R S p e c}\left(B_{m}\right)$ in the homotopy category of stacks, where $B_{m}$ is the CDGA whose underlying graded commutative algebra is freely generated by the symbols (6-4) and such that the differential is defined by (6-5).

Proposition 6.23 For $m \leq-2$, the CDGA $B_{m}$ is not homotopically finitely presented.

Proof In this range, $B_{m}$ is a minimal Sullivan algebra in the sense of Félix, Halperin and Thomas [11, Section II.12]. Let $B_{m, r} \subset B_{m}, r \geq 2$, be the subalgebra generated by the $x_{n}$ with $n \leq r$. Notice that the differential of $B_{m}$ restricts to $B_{m, r}$, so $B_{m, r}$ is actually a sub-CDGA of $B_{m}$. These sub-CDGA are also minimal Sullivan algebras. They define an increasing filtration of $B_{m}$ such that

$$
B_{m}=\bigcup_{r \geq 2} B_{m, r}=\operatorname{colim}_{r} B_{m, r}=\operatorname{hocolim}_{r} B_{m, r} .
$$


This is indeed a homotopy colimit since the inclusions $B_{m, r} \subset B_{m, r+1}$ are cofibrations of CDGAs.

If $B_{m}$ were homotopically finitely presented, the identity in $B_{m}$ would factor up to homotopy through the inclusion of a certain $B_{m, r} \subset B$. This factorization would induce a quasi-isomorphism from the linear part of $B_{m}$ to itself by [11, Proposition II.14.13]. This is impossible, since the linear part of $B_{m}$ is the unbounded graded $\mathbb{Q}$-module with basis $\left\{x_{n}\right\}_{n \geq 2}$ and trivial differential, and the linear part of $B_{m, r}$ is bounded. It is actually the subcomplex spanned by $\left\{x_{n}\right\}_{r \geq n \geq 2}$.

The stack Vect $n$ of rank $n$ vector bundles, $n \geq 0$, is 1 -geometric in the complicial algebraic geometry contexts since they satisfy the assumptions recalled in Example 5.20(1). We consider the substack $\underline{\operatorname{Vect}}_{n}[m] \subset \mathrm{QCoh}, m \in \mathbb{Z}$, such that, for any CDGA $A$, the connected components of $\underline{\operatorname{Vect}}_{n}[m](A)$ correspond to the $m$-fold suspensions of rank $n$ vector bundles. Obviously, suspension defines an isomorphism $\underline{\operatorname{Vect}}_{n}[m] \cong \underline{\operatorname{Vect}}_{n}$, $m \in \mathbb{Z}$, in the homotopy category of stacks, hence ${\underline{V^{\prime}}}_{n}[m]$ is 1 -geometric.

Corollary 6.24 For $m \leq-2$, the affine morphism between 1-geometric stacks

$$
\xi_{\underline{\text { Vect }_{1}[m]}}^{\text {Ass }_{1}}: \underline{\operatorname{Alg}_{\text {Vect }_{1}[m]}(\text { Ass }) \rightarrow \underline{\operatorname{Vect}}_{1}[m]}
$$

is not categorically locally finitely presented in the sense of [26, Definition 1.3.6.4 (1)].

Proof This follows from the fact that the homotopy pullback of $\xi_{\text {Vect }_{1}[m]}^{\text {Ass }}$ along the essentially unique morphism

$$
\mathbb{R} \underline{\operatorname{Spec}}(\mathbb{Q}) \rightarrow \underline{\operatorname{Vect}_{1}}[m],
$$

represented by $\Sigma^{m} \mathbb{Q}$, is the affine stack $\operatorname{Map}_{\mathrm{Op}(\mathrm{Ch}(\mathbb{Q}))}\left(\operatorname{Ass}_{\mathrm{Ss}} \operatorname{End}_{\mathrm{Ch}(\mathbb{Q})}\left(\Sigma^{m} \mathbb{Q}\right)\right)$, which is not homotopically finitely presented.

\section{Appendix A: The very strong unit axiom}

In this short appendix we consider a strengthening of Hovey's unit axiom and its consequences. Here, unlike in Definition 2.1 and the rest of the paper, monoidal model categories are not assumed to satisfy the strong unit axiom, but just Hovey's unit axiom.

Definition A.1 A monoidal model category $\mathscr{C}$ satisfies the very strong unit axiom if for any object $X$ and any cofibrant replacement $q: \widetilde{\mathbb{I}} \stackrel{\sim}{\rightarrow} \mathbb{I}$ of the tensor unit, the morphisms $X \otimes q$ and $q \otimes X$ are weak equivalences. 
This axiom has been implicitly used by Batanin and Berger in the proof of [4, Proposition 1.15]. The name 'very strong unit axiom' was coined by Berger in private communication.

Remark A.2 If the very strong unit axiom holds for a certain cofibrant resolution of $\mathbb{I}$ then it holds for any cofibrant resolution of $\mathbb{I}$. In particular, it is satisfied when $\mathbb{I}$ is cofibrant.

The strong unit axiom considered in [21, Definition A.9] is a weaker version where $X$ runs only over the pseudocofibrant objects. Recall that $X$ is pseudocofibrant if the functors $X \otimes-$ and $-\otimes X$ preserve cofibrations. This is equivalent to say that $X \otimes-$ and $-\otimes X$ are left Quillen functors; see [21, Remark A.2]. Cofibrant objects are pseudocofibrant by the pushout product axiom. The paradigm of pseudocofibrant object which need not be cofibrant is the tensor unit $\mathbb{I}$. Moreover, objects $X$ for which there exists a cofibration $\mathbb{I} \succ X$ are also pseudocofibrant; see [21, Remark B.2]. These objects are called $\mathbb{I}$-cofibrant.

The following three lemmas can be proved as [21, Lemmas A.11, A.12 and A.13], respectively, using the very strong unit axiom instead of the strong unit axiom.

Lemma A.3 Suppose that, for $q: \widetilde{\mathbb{I}} \stackrel{\sim}{\rightarrow} \mathbb{I}$ a cofibrant resolution of the tensor unit, the functors $\widetilde{\mathbb{I}} \otimes-$ and $-\otimes \widetilde{\mathbb{I}}$ preserve weak equivalences. Then the very strong unit axiom holds.

Remark A.4 In particular, the very strong unit axiom holds in monoidal model categories where cofibrant objects are flat. Recall that this means that the functors $X \otimes-$ and $-\otimes X$ preserve weak equivalences if $X$ is cofibrant.

The converse is also true, even something stronger holds.

Lemma A.5 If the very strong unit axiom holds, a morphism $f: U \rightarrow V$ is a weak equivalence if and only if $f \otimes \widetilde{\mathbb{I}}$ is a weak equivalence for some cofibrant replacement $\widetilde{\mathbb{I}}$ of the tensor unit. The same is true replacing $f \otimes \widetilde{\mathbb{I}}$ with $\widetilde{\mathbb{I}} \otimes f$.

The following result is used to show that module categories in a HAG context are algebras over the base symmetric monoidal model category.

Lemma A.6 Let $\mathscr{C}$ be a monoidal model category satisfying the very strong unit axiom. If $f: U \stackrel{\sim}{\rightarrow} V$ is a weak equivalence with pseudocofibrant source and target and $X$ is any object, then $f \otimes X$ and $X \otimes f$ are weak equivalences. 


\section{References}

[1] J Adámek, J Rosický, Locally presentable and accessible categories, London Math. Soci. Lecture Note Series 189, Cambridge Univ. Press (1994) MR1294136

[2] M Anel, Champs de modules des catégories linéaires et abéliennes, $\mathrm{PhD}$ thesis, Université Toulouse III - Paul Sabatier (2006)

[3] C Barwick, On left and right model categories and left and right Bousfield localizations, Homology, Homotopy Appl. 12 (2010) 245-320 MR2771591

[4] M Batanin, C Berger, Homotopy theory for algebras over polynomial monads arXiv: 1305.0086

[5] C Berger, I Moerdijk, Axiomatic homotopy theory for operads, Comment. Math. Helv. 78 (2003) 805-831 MR2016697

[6] F Borceux, Handbook of categorical algebra, 2, Encyclopedia of Mathematics and its Applications 51, Cambridge Univ. Press (1994) MR1313497

[7] D-C Cisinski, Invariance de la $K$-théorie par équivalences dérivées, J. K-Theory 6 (2010) 505-546 MR2746284

[8] W Crawley-Boevey, Exceptional sequences of representations of quivers, from: "Representations of algebras", CMS Conf. Proc. 14, Amer. Math. Soc. (1993) 117-124 MR1265279

[9] W G Dwyer, D M Kan, Function complexes in homotopical algebra, Topology 19 (1980) 427-440 MR584566

[10] W G Dwyer, D M Kan, A classification theorem for diagrams of simplicial sets, Topology 23 (1984) 139-155 MR744846

[11] Y Félix, S Halperin, J-C Thomas, Rational homotopy theory, Graduate Texts in Mathematics 205, Springer-Verlag, New York (2001) MR1802847

[12] P Gabriel, Finite representation type is open, from: "Proceedings of the International Conference on Representations of Algebras", (V Dlab, P Gabriel, editors), Carleton Math. Lecture Notes 9, Carleton Univ, Ottawa, ON (1974) MR0376769

[13] M Hovey, Model categories, Mathematical Surveys and Monographs 63, Amer. Math. Soc. (1999) MR1650134

[14] G Janelidze, G M Kelly, A note on actions of a monoidal category, Theory Appl. Categ. 9 (2001/02) 61-91 MR1897810

[15] G Laumon, L Moret-Bailly, Champs algébriques, Ergeb. Math. Grenzgeb. 39, Springer, Berlin (2000) MR1771927

[16] J Lurie, Higher algebra Available at http://www.math.harvard.edu/ lurie

[17] V Lyubashenko, O Manzyuk, Unital $A_{\infty}$-categories arXiv:0802.2885v1 
[18] S Mac Lane, Categories for the working mathematician, 2nd edition, Graduate Texts in Mathematics 5, Springer, New York (1998) MR1712872

[19] F Muro, Homotopy units in A-infinity algebras arXiv:1111.2723

[20] F Muro, Homotopy theory of nonsymmetric operads, Algebr. Geom. Topol. 11 (2011) 1541-1599 MR2821434

[21] F Muro, Homotopy theory of nonsymmetric operads, II, to appear in Algebr. Geom. Topol. (2014) arXiv:1304.6641

[22] C W Rezk, Spaces of algebra structures and cohomology of operads, PhD thesis, Mass. Inst. of Tech. (1996) Available at http://search.proquest.com//docview/ 304311860

[23] S Schwede, B E Shipley, Algebras and modules in monoidal model categories, Proc. London Math. Soc. 80 (2000) 491-511 MR1734325

[24] J D Stasheff, Homotopy associativity of $H$-spaces, I, II, Trans. Amer. Math. Soc. 108 (1963) 293-312 MR0158400

[25] B Toën, G Vezzosi, Brave new algebraic geometry and global derived moduli spaces of ring spectra, from: "Elliptic cohomology", (H R Miller, DC Ravenel, editors), London Math. Soc. Lecture Note Ser. 342, Cambridge Univ. Press (2007) 325-359 MR2330521

[26] B Toën, G Vezzosi, Homotopical algebraic geometry, II: Geometric stacks and applications, Mem. Amer. Math. Soc. 902 (2008) MR2394633

Universidad de Sevilla, Facultad de Matemáticas, Departamento de Álgebra Avda. Reina Mercedes s/n, 41012 Sevilla, Spain

fmuro@us.es

http://personal.us.es/fmuro

Received: 21 August 2013 
